\title{
Allele-selective lowering of mutant HTT protein by HTTLC3 linker compounds
}

$\mathrm{Li}, \mathrm{Z}$

http://hdl.handle.net/10026.1/15145

10.1038/s41586-019-1722-1

Nature

Springer Science and Business Media LLC

All content in PEARL is protected by copyright law. Author manuscripts are made available in accordance with publisher policies. Please cite only the published version using the details provided on the item record or document. In the absence of an open licence (e.g. Creative Commons), permissions for further reuse of content should be sought from the publisher or author. 
Zhaoyang $\mathrm{Li}^{1 \#}$, Cen Wang ${ }^{1 \#}$, Ziying Wang ${ }^{1 \#}$, Chenggang Zhu ${ }^{2 \#}$, Jie $\mathrm{Li}^{3}$, Tian Sha ${ }^{1}$, Lixiang Ma ${ }^{4}$, Chao $\mathrm{Gao}^{5}$, Yi Yang ${ }^{6}$, Yimin Sun ${ }^{1}$, Jian Wang ${ }^{1}$, Xiaoli Sun ${ }^{1}$, Chenqi Lu ${ }^{1}$, Marian Difiglia ${ }^{7}$, Yanai Mei ${ }^{1}$, Chen Ding ${ }^{1 \$}$, Shouqing Luo ${ }^{6 \$}$, Yongjun Dang ${ }^{8}$, Yu Ding ${ }^{1 *}$, Yiyan Fei ${ }^{2 *}$, Boxun $\mathrm{Lu}^{1^{*}}$

${ }^{1}$ Neurology Department at Huashan Hospital, State Key Laboratory of Medical Neurobiology and MOE Frontiers Center for Brain Science, Institutes of Brain Science, School of Life Sciences, Fudan University, Shanghai, China.

${ }^{2}$ Department of Optical Science and Engineering, Shanghai Engineering Research Center of Ultra-Precision Optical Manufacturing, Key Laboratory of Micro and Nano Photonic Structures (Ministry of Education), Fudan University, Shanghai, China.

${ }^{3}$ National Facility for Protein Science in Shanghai, Large-scale Preparation System, Shanghai, China.

${ }^{4}$ Department of Anatomy, Histology and Embryology, Shanghai Medical College, Fudan University, Shanghai, China.

${ }^{5}$ Institutes of Biomedical Sciences, Fudan University, Shanghai, China.

${ }^{6}$ Peninsula Schools of Medicine and Dentistry, Institute of Translational and Stratified Medicine, University of Plymouth, Plymouth, UK.

${ }^{7}$ Laboratory of Cellular Neurobiology, Department of Neurology, Massachusetts General Hospital, Charlestown, Massachusetts, United States of America

${ }^{8}$ Key Laboratory of Metabolism and Molecular Medicine, Shanghai Medical College, Fudan University, Shanghai, China.

\# These authors contribute equally;

"Corresponding authors:

Boxun Lu (lead contact): luboxun@fudan.edu.cn

Yiyan Fei: fyy@fudan.edu.cn

Yu Ding: yuding@fudan.edu.cn

${ }^{\$}$ Co-senior authors 


\section{Summary}

Accumulation of mutant proteins is the major cause for many diseases (proteopathies), and lowering the level of these proteins is highly desired for treatment. We hypothesized that compounds interacting with both the autophagosome protein LC ${ }^{1}$ and the disease-causing protein may target the latter for autophagic clearance. We tested this hypothesis in the context of lowering mutant HTT protein (mHTT), which contains an expanded polyglutamine (polyQ) tract and causes Huntington's disease (HD), an incurable neurodegenerative disorder ${ }^{2}$. Through small-molecule-microarray based screening, we identified four compounds interacting with both LC3 and mHTT, but not the wild-type HTT protein (wtHTT). Some of these compounds targeted mHTT to autophagosomes, reduced mHTT levels in an allele-selective manner, and rescued HD-relevant phenotypes in cells and in vivo in the fly and mouse HD models. We further revealed that these compounds interacted with the expanded polyQ stretch and could lower the level of mutant ATXN3, another disease-causing protein with expanded poly $Q^{3}$. Our study provides candidate compounds for lowering mHTT and potentially other disease-causing proteins with polyQ expansion, demonstrating the concept of lowering disease-causing proteins by autophagosome-tethering compounds (ATTEC). 


\section{Background}

An emerging approach for disease treatment is to lower the levels of disease-causing proteins, especially those with unknown activities. Biological tools such as RNAi or CRISPR may achieve this goal $^{4-6}$, but their clinical delivery is challenging. Enhancing proteasomal degradation of target proteins by "PROTAC" is a promising emerging approach ${ }^{7}$, but proteasomes alone are inefficient in degrading certain large proteins or aggregates ${ }^{8}$. Another independent protein degradation pathway is macroautophagy (referred to as autophagy hereafter), which is a bulk degradation system that engulfs proteins into autophagosomes for subsequent lysosomal degradation ${ }^{9}$. Autophagy is present in all eukaryotic cells, and thus harnessing the power of autophagy to degrade certain target proteins may open new windows for drug discovery. Here we investigate this possibility in the context of lowering $\mathrm{mHTT}$, which contains an expanded polyglutamine (polyQ) stretch ( $\geq 36 \mathrm{Q})$ and causes HD, an incurable monogenetic neurodegenerative disorder ${ }^{2}$. mHTT could be degraded by autophagy, during which protein substrates are engulfed into double-membrane autophagosomes associated with lipidated LC ${ }^{1}$. We thus hypothesized that linker compounds interacting with both mHTT and LC3 may tether them together to enhance the recruitment of $\mathrm{mHTT}$ into autophagosomes, facilitating mHTT degradation. In addition, MHTT-LC3 linker compounds that do not interact with wtHTT may promote allele-selective degradation of mHTT. Since no mHTT/LC3-interacting compounds have been reported, we performed small-molecule-microarray-based screening for desired compounds, and utilized 
wtHTT for the counter-screen to identify allele-selective candidates.

\section{Results}

\section{Identification of mHTT-LC3 linker compounds}

We stamped 3375 compounds (Fig. 1a) in duplicates into a microarray on isocyanate-functionalized glass slides via the nucleophile-isocyanate reaction, which forms covalent bonds between the compounds and the glass slides ${ }^{10,11}$. We then purified the human LC3B protein ${ }^{1}$ (Extended Data Fig. 1a-b \& Supplementary Table 1), a pathogenic mHTT exon1 fragment ${ }^{12}$ with expanded polyQ (mHTTexon1-Q72), and a control wtHTT exon1 fragment (HTTexon1-Q25) (Extended Data Fig. 1c-d) for the screen. We fused a maltose-binding-protein (MBP) tag to both HTT exon1 proteins to increase their solubility required for later experiments.

To identify LC3B- and mHTT- interacting compounds, we had these purified proteins flow through the SMMs, and detected the compound-protein interaction using an optical biosensor, the scanning oblique-incidence reflectivity difference (OI-RD) microscope. OI-RD is a widely used interaction measurement technology ${ }^{13-15}$, whose working principle has been validated and reported previously ${ }^{16-18}$. We then performed experiments with HTTexon1-Q25 or buffer alone to exclude non-specific signals, and identified two compounds that interact with both LC3B and mHTTexon1-Q72, but not HTTexon1-Q25: 1005 (GW5074, 3-3-[(3,5-Dibromo-4-hydroxyphenyl)methylidene]-5-iodo-1H-indol-2-one) and 8F20 (ispinesib, N-(3-aminopropyl)-N-[(1R)-1-[7-chloro-4-oxo-3-(phenylmethyl)-2-quinazolinyl]-2-meth 
ylpropyl]-4-methylbenzamide) (Fig. 1b, annotated based on their ID in the compound library). We then measured the $\mathrm{K}_{\mathrm{on}}$ and $\mathrm{K}_{\text {off }}$ of these interactions to confirm our observation (Fig. 1c-d) and revealed $\sim 100 \mathrm{nM} \mathrm{K}_{\mathrm{d}}$ of the compounds' interaction with LC3B or mHTTexon1-Q72. As shown in Fig. 1e, these compounds also interacted with the full-length mHTT (fIHTT-Q73, Extended Data Fig. 1e), but not wtHTT (HTTexon1-Q25 or flHTT-Q23, Fig. 1c\&e) or irrelevant proteins (Extended Data Fig. 2a) including MBP-His8 (MBP), superfolder GFP (sfGFP), and Rpn10 (a proteasomal ubiquitin-receptor) (Extended Data Fig. 1f). We then validated the interaction by an orthogonal assay, microscale thermophoresis (MST), and obtained consistent results (Extended Data Fig. 2b).

mHTT-LC3 linker compounds reduced mHTT levels via autophagy in an allele-selective manner

We then tested if these potential mHTT-LC3 linker compounds decrease mHTT levels via autophagy as predicted. Both hits decreased levels of MHTT in cultured primary cortical neurons from a well-established HD knockin mouse model $\left(\mathrm{Hdh}^{\mathrm{Q7/Q140}}\right)^{19}$ (Fig. 2a), while had little or no effect on levels of wtHTT in the heterozygous HD neurons ( $\mathrm{Hdh}^{\mathrm{Q7/Q140}}$ ) (Fig. 2a) and wild-type neurons ( $\left.\mathrm{Hdh}^{\mathrm{Q7/Q7}}\right)$ (Fig. $2 b)$, consistent with their lack of wtHTT interaction. We then looked for other mHTT-LC3 linker compounds based on the common features of the two hit compounds 1005 and $8 \mathrm{~F} 20$. The $-\mathrm{OH}$ group in 1005 and the $-\mathrm{NH}_{2}$ group in $8 \mathrm{~F} 20$ were utilized in the nucleophile-isocyanate reaction for stamping of the SMMs, and these groups were inaccessible to $\mathrm{mHTT}$ and LC3B for the compound-protein 
115 interaction during the screening (Fig. 2c). Thus, while these two hit compounds have

116 different structures, their exposed chemical groups on the SMMs shared similarities

117 by containing an aryl ring connected with a lactam-based bicyclic structure with

118 halogen-substituted aryl group (Fig. 2c). We tested several compounds with similar

119 features and identified two additional mHTT-LC3 linker compounds (Fig. 2c, AN1

120 (3-5-Bromo-3-[(3-bromo-4,5-dihydroxyphenyl)methylidene]-1H-indol-2-one) and AN2

121 (5,7-Dihydroxy-4-phenylcoumarin), for analog1 and analog2, respectively) that

122 interact with both mHTT and LC3B, but not wtHTT or irrelevant control proteins

123 (Extended Data Fig. 2c-d). They also reduced the levels of mHTT in an

124 allele-selective manner in cultured HD mouse neurons (Fig. 2d). No cytotoxicity was

125 observed in cultured neurons treated with these compounds at the tested

126 concentration range (Extended Data Fig. 2e), confirming that the mHTT lowering was

127 not due to cell loss.

128 Most of these compounds showed an optimal dose (hook effect) in lowering

$129 \mathrm{mHTT}$ (Fig. 2a\&d): a sufficient concentration is desired for tethering $\mathrm{mHTT}$ and LC3

130 together, but excessively high concentrations may cause the compound molecules to

131 interact with mHTT and LC3 separately, without tethering them. Similar concentration

132 dependent effects were observed in HD patient fibroblasts (Fig. 3c, right panel) and

133 have been reported for PROTAC ${ }^{20}$. Consistent with the prediction that the $\mathrm{mHTT}$

134 lowering is mediated by degradation via autophagy, the autophagy inhibitor $\mathrm{NH}_{4} \mathrm{Cl}$ or

135 chloroquine blocked the mHTT-lowering effects (Extended Data Fig. 3a), confirming

136 that the compounds targeted mHTT for autophagic degradation. Meanwhile, the 
compound-induced mHTT-lowering effects were only slightly enhanced by the mTOR inhibitor rapamycin, an enhancer of autophagosome formation (Extended Data Fig. 3a, right panels; also see Fig. 3b).

The mHTT lowering could be detected by multiple mHTT antibodies, including 3B5H10, which detects a toxic species of the expanded polyQ stretch ${ }^{21,22}$ (Fig. 2d, right panel), suggesting that the detected $\mathrm{mHTT}$ lowering was not due to affinity changes to a specific antibody. In addition, we did not observe any obvious increase of possible polyQ-containing mHTT fragments at lower molecular weights (Extended Data Fig. 3b-c), suggesting that the mHTT lowering was not due to increased site-specific cleavages of mHTT.

$$
\text { We further investigated the compounds' effects in HD patient cells by the }
$$
well-established HTRF (Homologous Time-Resolved Fluorescence) assay ${ }^{23,24}$, which is more quantitative than Western-blots, although inapplicable to mouse $\mathrm{mHTT}$ proteins due to non-specific signals ${ }^{25}$. We observed autophagy-dependent lowering of mHTT by these compounds in HD patient fibroblasts and iPSC-derived neurons (Fig. 3a-b\&Extended Data Fig. 3d), but not wtHTT in the wild-type or Parkinson's disease (PD) patient fibroblasts (Fig. 3a). To further confirm the role of autophagic degradation, we tested the compounds' effect with or without lowering of ATG5, a key autophagy gene required for autophagosome formation ${ }^{26}$. ATG5 knockdown in HD patient fibroblasts (Q47) significantly decreased LC3-II and nullified the MHTT lowering effects induced by the mHTT-LC3 linker compounds (Extended Data Fig. 3e). Similar results were obtained in ATG5 knockout mouse embryonic fibroblasts ${ }^{26}$ (MEFs, 
Extended Data Fig. 3f), confirming that the compounds' effects were mediated by autophagic degradation.

The two hit compounds 1005 and 8F20 are known to inhibit c-Raf and $\mathrm{KSP}^{27,28}$, respectively, whereas AN1 and AN2 had unknown activities on these targets. We thus tested their potential influences on c-Raf and KSP. Based on the in vitro c-Raf kinase assay, 1005, the known c-Raf inhibitor, but not the other three compounds inhibited c-Raf at the concentrations tested (Extended Data Fig. 4a). We then tested Mek/Erk phosphorylation levels in the cultured neurons treated with these compounds at optimal mHTT-lowering concentrations to evaluate Raf activity ${ }^{29}$ and found no significant effects of all tested compounds (Extended Data Fig. 4b, left panel). We also tested phospho-BUBR1 levels to evaluate KSP activities ${ }^{30}$, and observed no significant effects either (Extended Data Fig. 4b, right panel). We made similar observations in the HD patient fibroblasts (Q47) (Extended Data Fig. 4c). Thus, the observed mHTT-lowering is probably irrelevant to c-Raf or KSP inhibition. To further confirm this, we examined the effects of several known c-Raf or KSP inhibitors, and found that they had no HTT-lowering effects (Fig. 3c, left panel). Two of these inhibitors, PLX-4720 and BAY1217389, have structures somewhat similar to 1005 and 8 F20, respectively (Fig. 3c, middle panel). They showed no effects in lowering mHTT in patient cells at sub-micromolar concentrations (Fig. 3c, right panel), probably because they had very weak affinity to LC3 and mHTT, if any (Extended Data Fig. 2c, right panels). In comparison, AN2 showed a dose-dependent mHTT lowering in the same cells (Fig. 3c, right panel). 
homolog Atg8 has a predicted structure highly similar to LC3B (Extended Data Fig.

5a), we tested the compounds in HD transgenic flies expressing human full-length

mHTT. All of the discovered mHTT-LC3 linker compounds significantly reduced $\mathrm{mHTT}$

levels (Extended Data Fig. 5b), validating the in vivo efficacy of these compounds.

We further investigated the compounds' effects in vivo in the HD knockin mouse

model ( $\left.\mathrm{Hdh} \mathrm{Q}^{\mathrm{Q} / \mathrm{Q} 140}\right)^{19}$ by intracerebroventricular (icv) injections. Three out of the four

linker compounds (1005, AN1 or AN2, but not 8F20) led to significant lowering of

mHTT in cortices of HD mice (Extended Data Fig. 6a). We then performed

intraperitoneal (ip) injection of 1005 and AN2 at $0.5 \mathrm{mg} / \mathrm{kg}$ in HD knockin mice. The

compounds crossed the blood-brain barrier and reached the brain at detectable

concentrations (Extended Data Fig. 5c, $\sim 20$ to $200 \mathrm{nM}$ for 1005 and $\sim 20$ to $40 \mathrm{nM}$ for

AN2; no signal was detected in the DMSO injected control group) $0.5 \sim 6$ hours after

injection. Consistent with this, we observed significant allele-selective lowering of

mHTT in mouse cortices and striata (Extended Data Fig. 6b-c). The observed

lowering was not due to changes in mHTT solubility, because no increase of mHTT

aggregates was observed in the cortical tissues of mice treated with these

compounds (Extended Data Fig. 6d).

mHTT-LC3 linker compounds tethered mHTT to autophagosomes

We then examined whether these compounds truly function as linkers between

AN2, the two compounds that were effective in vivo by ip-injection, markedly 
enhanced the mHTT-LC3 interaction in the in vitro pull-down experiments (Fig. 4a, comparing lane 11 with $12 \& 13$; Fig. 4b, comparing lane 11 with 12 for both upper and lower panels). No enhancement effect was observed for the wtHTT-LC3 interaction (Fig. 4a, lane 8-10; Fig. 4b, lane 9-10 in both upper and lower panels). Consistent with this, these compounds led to elevated engulfment of $\mathrm{mHTT}$ by autophagosomes both in transiently transfected HeLa cells expressing exogenous GFP-LC3B and HTTexon1-MBP-His fragments (Fig. 4c), and in mouse striatal cells $\left(\text { STHdh }{ }^{\text {Q111/Q111 }}\right)^{31}$ expressing endogenous LC3 and full-length mHTT proteins (Fig. 4d).

The data above confirmed that the compounds tethered mHTT and LC3B/autophagosomes in vitro and in cells, although the detailed structural information remains to be resolved.

mHTT-LC3 linker compounds did not influence autophagy function and reduced mHTT in a relatively specific manner

$$
\text { The mHTT-lowering by the linker compounds was unlikely due to an }
$$
enhancement of autophagy functions, because the number and size of autophagosomes remained unchanged (Extended Data Fig. 7a). We then further investigated whether the compounds could influence autophagy by established approaches in the literature ${ }^{32-34}$. Neither 1005 nor AN2 influenced the autophagosome-lysosome fusion or autophagy activity (Extended Data Fig. 7b-d). Furthermore, we observed no changes of the LC3-II level in the cultured cortical neurons treated with 1005 or AN2 in the absence or presence of the lysosome inhibitor bafilomycin-A1 (bafA1) (Extended Data Fig. 7e). The level of the known 
autophagy-selective substrate protein SQSTM1/p62 was also unaffected in vivo and in cultured neurons (Extended Data Fig. 7f \& 8a). In addition, other wild-type polyQ proteins (Atxn3 and Tbp) and control proteins (Nbr1, Ncoa4, Actin, Gapdh, Tubulin) were not influenced (Extended Data Fig. 8a, all changes $<10 \%$ ).

We then performed proteomics analysis to obtain a more complete scope of proteins that may have been influenced. We observed significant lowering $(\sim 20 \%, p<$ 0.01) of HTT levels in cortices of mice ip-injected with 1005 or AN2 (Extended Data Fig. $8 \mathrm{~b}$, bar plots). Considering that the proteomics analysis was unable to distinguish mHTT from wtHTT, the actual mHTT lowering was expected to be more. Meanwhile, using the criteria of $p<0.01$, we observed changes of only a small percentage of proteins (Extended Data Fig. 8b, and see Supplementary Table 2 for details). No autophagy-specific substrate proteins exhibited significant changes and there was no enrichment of autophagy pathway-related proteins (Supplementary Table 2), further confirming that autophagy was unaffected. Proteomics analysis in cultured neurons revealed consistent results (Extended Data Fig. 8c, and see Supplementary Table 3 for details).

mHTT-LC3 linker compounds lowered other proteins with expanded polyQ

The discovered linker compounds interacted with and lowered mHTT but not wtHTT (Fig. 1). The simplest explanation is that the compounds specifically interact with the expanded polyQ tract, possibly by recognizing its emergent conformation that is different from the short polyQ stretch ${ }^{21,35}$. If so, the discovered linker compounds may reduce other proteins with expanded polyQ. Consistent with this prediction, the 
247 linker compounds 10O5, AN1 and AN2 reduced the levels of mutant but not wild-type

248 ATXN3 in SCA3 patient fibroblasts (Extended Data Fig. 9a) and exogenously

249 expressed 72Q-GFP, 46Q-GFP, 38Q-GFP but not 25Q-GFP proteins (containing just

250 the Met-polyQ-sfGFP sequences) in HEK293T cells (Extended Data Fig. 9b). These

251 data suggest that the compounds distinguished the expanded from the short polyQ

252 stretch at a threshold between $25 \mathrm{Q}$ and $38 \mathrm{Q}$. To further confirm this, we tested the

253 compound-polyQ interactions (Extended Data Fig. 9c), and confirmed that 1005, AN1

254 and AN2 interacted with polyQ-GFP with $38 \mathrm{Q}$ or longer polyQ, but not $25 \mathrm{Q}-\mathrm{GFP}$ or

255 GFP alone (Extended Data Fig. 9d-e; Extended Data Fig. 2).

256

mHTT-LC3 linker compounds rescued HD-relevant phenotypes

257 We further investigated the therapeutic potential of the compounds in treating HD.

258 All the mHTT-LC3 linker compounds rescued mHTT toxicity in HD patient

259 iPSC-derived neurons (Fig. 5a-b). They also rescued HD-relevant behavioral deficits

260 and increased the lifespan of flies expressing human mHTT, while having no influence

261 on the flies expressing wtHTT (Fig. 5c).

262 Finally, we investigated the disease-relevant behavioral phenotypes in 10-months

263 old heterozygous HD knockin mouse $\left(\mathrm{Hdh}^{\mathrm{Q7/Q140}}\right)$. Significant deficits of HD mice were

264 observed in several behavioral tests including rotarod, balance beam, and gripping

265 force tests, while the DMSO $(110 \mu \mathrm{g} / \mathrm{kg})$ injection alone did not have an effect

266 (Extended Data Fig. 9f-h). In comparison, ip injection of the 1005 or AN2 significantly

267 improved HD-relevant behavioral deficits in these tests, without influencing the

268 wild-type mice (Fig. 5d-f), demonstrating a rescue of HD-relevant phenotypes. This is 
a proof of principle study, and further investigations will be required to establish the

270 suitability for therapeutic application.

\section{Discussion}

272 In this study, we have identified mHTT-LC3 linker compounds that were able to

273 reduce $\mathrm{mHTT}$ levels at $\sim \mathrm{nM}$ concentrations in $\mathrm{HD}$ cells and at $0.5 \mathrm{mg} / \mathrm{kg}$ by ip

274 injection in vivo (Extended Data Table 1). The compounds did not influence wtHTT,

275 which has essential functions especially during development and young adulthood ${ }^{36}$.

276 These features of the compounds are highly desired for HD treatment or potentially

277 other polyQ diseases (Extended Data Fig. 9a-e), although preclinical studies of

278 longitudinal efficacy and safety are necessary for therapeutic development.

279 From a broader perspective, we demonstrate the concept of using small molecule

280 compounds to "glue" the target protein (mHTT) and LC3 for autophagic degradation

281 (Fig. 5g). We selected mHTT as our target protein, because wtHTT provides a perfect

282 control for screening. We had the fortune to discover hits that interact with both LC3B

283 and mHTT. If no such hits had been identified, linker compounds could still be

284 generated by conjugating an mHTT-interacting compound and an LC3-interacting

285 compound by the nucleophile-isocyanate reaction utilized for the stamping of SMMs.

286 To develop this concept, the critical next step is to resolve the core chemical

287 compartment that interacts with LC3 without influencing its function. Comprehensive

288 medicinal chemistry and structural studies are needed to resolve the compound-LC3

289 interaction interface, which may then be developed to a general degradation tool for

290 conjugation with other compounds interacting with specific targets of interest. 

of utilizing autophagosome-tethering compounds (ATTEC) to lower target proteins,

294 providing new entry points for drug discovery.

295

296

297

298

299

300

301

302

303

304

305

306

307

308

309

310

311

312

313

314

315

316

317

318

319

320

321

322

323

324

325

326

327

328

329

\section{Reference}

1 Kabeya, Y. et al. LC3, A mammalian homologue of yeast Apg8p, is localized in autophagosome membranes after processing. Embo J 19, 5720-5728, doi: 10.1093/emboj/19.21.5720 (2000).

2 Scherzinger, E. et al. Huntingtin-encoded polyglutamine expansions form amyloid-like protein aggregates in vitro and in vivo. Cell 90, 549-558, doi: 10.1016/s0092-8674(00)80514-0 (1997).

3 Warrick, J. M. et al. Expanded polyglutamine protein forms nuclear inclusions and causes neural degeneration in Drosophila. Cell 93, 939-949, doi:10.1016/s0092-8674(00)81200-3 (1998).

$4 \quad$ Fire, A. et al. Potent and specific genetic interference by double-stranded RNA in Caenorhabditis elegans. Nature 391, 806-811, doi:10.1038/35888 (1998).

5 Mali, P. et al. RNA-guided human genome engineering via Cas9. Science 339, 823-826, doi:10.1126/science.1232033 (2013).

6 Cong, L. et al. Multiplex genome engineering using CRISPR/Cas systems. Science 339, 819-823, doi:10.1126/science.1231143 (2013).

7 Winter, G. E. et al. Phthalimide conjugation as a strategy for in vivo target protein degradation. Science 348, 1376-1381, doi:10.1126/science.aab1433 (2015).

8 Lu, K., den Brave, F. \& Jentsch, S. Pathway choice between proteasomal and autophagic degradation. Autophagy 13, 1799-1800, doi:10.1080/15548627.2017.1358851 (2017).

9 Mizushima, N., Levine, B., Cuervo, A. M. \& Klionsky, D. J. Autophagy fights disease through cellular self-digestion. Nature 451, 1069-1075, doi:10.1038/nature06639 (2008).

10 Zhu, C. et al. Developing an efficient and general strategy for immobilization of small molecules onto microarrays using isocyanate chemistry. Sensors (Basel) 16, doi:10.3390/s16030378 (2016).

11 Fei, Y. et al. Screening small-molecule compound microarrays for protein ligands without fluorescence labeling with a high-throughput scanning microscope. J Biomed Opt 15, 016018, doi:10.1117/1.3309743 (2010).

12 Mangiarini, L. et al. Exon 1 of the HD gene with an expanded CAG repeat is sufficient to cause a progressive neurological phenotype in transgenic mice. Cell 87, 493-506, doi:S0092-8674(00)81369-0 (1996). 
13 Liu, $H$. et al. Nuclear cGAS suppresses DNA repair and promotes tumorigenesis. Nature 563, 131-136, doi:10.1038/s41586-018-0629-6 (2018). Landry, J. P. et al. Discovering small molecule ligands of vascular endothelial growth factor that block VEGF-KDR binding using label-free microarray-based assays. Assay Drug Dev Technol 11, 326-332, doi:10.1089/adt.2012.485 (2013).

15 Fei, Y. et al. Characterization of receptor binding profiles of influenza A viruses using an ellipsometry-based label-free glycan microarray assay platform. Biomolecules 5, 1480-1498, doi:10.3390/biom5031480 (2015).

340

Zhu, X. et al. Oblique-incidence reflectivity difference microscope for label-free high-throughput detection of biochemical reactions in a microarray format. Appl Opt 46, 1890-1895, doi:10.1364/ao.46.001890 (2007).

Landry, J. P., Zhu, X. D. \& Gregg, J. P. Label-free detection of microarrays of biomolecules by oblique-incidence reflectivity difference microscopy. Opt Lett 29, 581-583, doi:10.1364/ol.29.000581 (2004).

349

Zhu, C. et al. Fast focal point correction in prism-coupled total internal reflection scanning imager using an electronically tunable lens. Sensors (Basel) 18, doi:10.3390/s18020524 (2018).

358

359

Menalled, L. B., Sison, J. D., Dragatsis, I., Zeitlin, S. \& Chesselet, M. F. Time course of early motor and neuropathological anomalies in a knock-in mouse model of Huntington's disease with 140 CAG repeats. J Comp Neurol 465, 11-26, doi:10.1002/cne.10776 (2003).

360

361

362

Bondeson, D. P. et al. Catalytic in vivo protein knockdown by small-molecule PROTACs. Nat Chem Biol 11, 611-617, doi:10.1038/nchembio.1858 (2015).

363

364

365

Miller, J. et al. Identifying polyglutamine protein species in situ that best predict neurodegeneration. Nat Chem Biol 7, 925-934, doi:10.1038/nchembio.694 (2011).

366

367

$\mathrm{Fu}, \mathrm{Y}$. et al. A toxic mutant huntingtin species is resistant to selective autophagy. Nat Chem Biol 13, 1152-1154, doi:10.1038/nchembio.2461 (2017). Baldo, B. et al. TR-FRET-based duplex immunoassay reveals an inverse correlation of soluble and aggregated mutant huntingtin in huntington's disease. Chem Biol 19, 264-275, doi:10.1016/j.chembiol.2011.12.020 (2012). human tissues: a bioassay for Huntington's disease. Anal Biochem 395, 8-15, doi:10.1016/j.ab.2009.08.001 (2009).

Lu, B. et al. Identification of NUB1 as a suppressor of mutant Huntington toxicity via enhanced protein clearance. Nat Neurosci 16, 562-570, doi:10.1038/nn.3367 (2013).

26 Mizushima, N. et al. Dissection of autophagosome formation using Apg5-deficient mouse embryonic stem cells. J Cell Biol 152, 657-668, doi:10.1083/jcb.152.4.657 (2001).

Lackey, K. et al. The discovery of potent cRaf1 kinase inhibitors. Bioorg Med Chem Lett 10, 223-226, doi:10.1016/s0960-894x(99)00668-x (2000).

Reddy, K. \& D'Orazio, A. Highlights from the international conference on 
molecular targets and cancer therapeutics: discovery, biology, and clinical applications, Philadelphia, PA. ECCO 13--The European Cancer Conference, Paris, France, October 30-November 3, 2005. Clin Genitourin Cancer 4, 156-159, doi:10.1016/S1558-7673(11)70136-7 (2005).

29 Johnson, G. L. \& Lapadat, R. Mitogen-activated protein kinase pathways mediated by ERK, JNK, and p38 protein kinases. Science 298, 1911-1912, doi:10.1126/science.1072682 (2002).

30 Tao, W. et al. An inhibitor of the kinesin spindle protein activates the intrinsic apoptotic pathway independently of p53 and de novo protein synthesis. Mol Cell Biol 27, 689-698, doi:10.1128/MCB.01505-06 (2007).

31 Trettel, F. et al. Dominant phenotypes produced by the HD mutation in STHdh(Q111) striatal cells. Hum Mol Genet 9, 2799-2809, doi:10.1093/hmg/9.19.2799 (2000).

32 Kimura, S., Noda, T. \& Yoshimori, T. Dissection of the autophagosome maturation process by a novel reporter protein, tandem fluorescent-tagged LC3. Autophagy 3, 452-460, doi:10.4161/auto.4451 (2007).

33 Zhang, J., Wang, J., Ng, S., Lin, Q. \& Shen, H. M. Development of a novel method for quantification of autophagic protein degradation by AHA labeling. Autophagy 10, 901-912, doi:10.4161/auto.28267 (2014).

$34 \mathrm{Ni}, \mathrm{H}$. M. et al. Dissecting the dynamic turnover of GFP-LC3 in the autolysosome. Autophagy 7, 188-204, doi:10.4161/auto.7.2.14181 (2011).

35 Feng, X., Luo, S. \& Lu, B. Conformation polymorphism of polyglutamine Proteins. Trends Biochem Sci 43, 424-435, doi:10.1016/j.tibs.2018.03.002 (2018).

36 Wang, G., Liu, X., Gaertig, M. A., Li, S. \& Li, X. J. Ablation of huntingtin in adult neurons is nondeleterious but its depletion in young mice causes acute pancreatitis. Proc Natl Acad Sci $U$ S A 113, 3359-3364, doi:10.1073/pnas.1524575113 (2016).

37 Vijayvargia, R. et al. Huntingtin's spherical solenoid structure enables polyglutamine tract-dependent modulation of its structure and function. Elife $\mathbf{5}$, e11184, doi:10.7554/eLife.11184 (2016). 
Main figure legends

Figure 1. Identification of potential mHTT-LC3 linker compounds by SMM-based screening and validation

a) The OI-RD image of a small molecule microarray (SMM). Each compound was printed in duplicates in adjacent vertical positions.

b) Magnified view of surface mass density changes after incubation with HTTexon1-Q25-MBP, HTTexon1-Q72-MBP, and LC3B, respectively. Two hits (1005 and $8 \mathrm{~F} 20$ ) were highlighted by red rectangular boxes.

c-e) Association-dissociation curves of surface immobilized compounds 8F20 and 1005 with HTTexon1-Q72-MBP (Q72), HTTexon1-Q25-MBP (Q25), LC3B, full-length HTT-Q73 (Q73), or full-length HTT-Q23 (Q23) at the indicated concentrations of purified proteins. For details see "Methods".

Figure 2. mHTT-LC3 linker compounds lowered mHTT but not wtHTT through autophagy in cultured mouse neurons.

a) Western-blots (HTT detected by the 2166 antibody) and quantifications of compound-treated cultured cortical neurons from $\mathrm{Hdh}^{\mathrm{Q7/Q140}} \mathrm{HD}$ knockin mice. The statistical analysis was performed by two-way ANOVA tests. For 1005, $F(1,72)=$ 50.93, $p<0.0001$; for 8F20, $F(1,40)=8.903, p=0.0048$.

b) Representative Western-blots (from 3 biological repeats) of cultured wild-type cortical neurons treated with the indicated compounds.

c) 2D-structures of the hit compounds and the other effective linker compounds identified. The red lines indicate the glass-chips used to stamp the hit compounds. The dotted ovals indicate the possible chemical groups exposed for protein-compound interactions in the screening.

d) Left and middle panels: similar to a), but treated with the compounds AN1 or AN2. For AN1, $F(1,70)=32.96, p<0.0001$; For AN2, $F(1,69)=23.03, p<0.0001$. Right panels: similar to a), but blotted with different HTT antibodies (1005 and 8F20: 100nM; AN2: $50 \mathrm{nM}$ ).

For all panels, $\mathrm{n}$ indicates the number of independently plated wells, and bars represent mean and s.e.m.. Full-blots of cropped gels are shown in Extended Data Fig. 3b or Supplementary Fig. 1.

Figure 3. mHTT-LC3 linker compounds lowered $\mathrm{mHTT}$ in HD patient cells.

a) HTT levels measured by HTRF (2B7/MW1 for mHTT, and 2B7/2166 for total HTT) in the indicated HD, WT or PD patient primary fibroblasts treated with the indicated compounds (100 nM). All signals were normalized to the averaged signals from the DMSO control group. The statistical analysis was performed by one-way ANOVA with post-hoc Dunnett's tests. “****: $p<0.0001$.

b) Similar to a), but in immortalized HD patient fibroblasts treated with or without the autophagy inhibitors $\mathrm{NH}_{4} \mathrm{Cl} /$ chloroquine/bafilomycin-A1, or the autophagy enhancer rapamycin.

c) Left panel: similar to a), but in immortalized HD patient fibroblasts (Q47) treated with indicated c-Raf or KSP inhibitors at $100 \mathrm{nM}$. Middle panel: 2D structure of 
450

451

452

453

454

455

456

457

458

459

460

461

462

463

464

465

466

467

468

469

470

471

472

473

474

475

476

477

478

479

480

481

482

483

484

485

inhibitors. The dotted ovals indicate the parts of the compounds that share similarities with the hit compounds. Right panel: dose-dependent curves of the indicated compounds.

For all panels, $\mathrm{n}$ indicates the number of independently plated wells, and bars represent mean and s.e.m..

Figure 4. Linker compounds enhanced mHTT-LC3 interaction and tethered mHTT to autophagosomes

a-b) Representative results (from 3 biological repeats) of in vitro pull-down experiments using purified HTT and LC3B proteins. For details see "Methods". c-d) Representative images (scale bar: $10 \mu \mathrm{m}$ ) and quantifications of the co-localization between HTT and autophagosomes. For details see "Methods". Bars present mean and s.e.m.. The n number indicates the number of cells. The statistical analysis was performed by one-way ANOVA with post-hoc Dunnett's tests. “****”: $p<0.0001$.

Figure 5. Linker compounds rescued HD-relevant phenotypes in cells and in vivo.

a) Representative immunostaining images (scale bar: $50 \mu \mathrm{m}$ ) and quantifications of the neuronal specific tubulin marker TUBB3 and DAPI staining showing neuronal morphology of patient iPSC-derived striatal neurons (HD: Q47; WT: Q19) treated with indicated compounds. For details see "Methods".

b) Neuronal apoptosis measurement at different time points after BDNF removal using a green fluorescent dye (NucView 488) detecting active caspase-3. For details see "Methods".

c) Left: Kaplan-Meier survival curves of transgenic Drosophila with the indicated transgenes and compound treatments. Right: similar to the left panel, but plotting the climbing performance as a function of age after eclosion. For details see "Methods". d-f) Mouse behavioral tests showing the improvement of HD-relevant phenotypes by ip-injection of the indicated compounds at $0.5 \mathrm{mg} / \mathrm{kg}$. For details see "Methods".

g) A schematic model showing how mHTT-LC3 linker compounds may induce mHTT degradation, illustrating the concept of lowering target proteins by autophagosome-tethering compounds (ATTEC). The images representing HTT proteins were published previously ${ }^{37}$. 
486

487

488

489

490

491

492

493

494

495

496

497

498

499

500

501

502

503

504

505

506

507

508

509

510

511

512

513

514

515

516

517

518

519

520

521

522

Methods

\section{Additional details of figure legends}

Figure 1. Identification of potential mHTT-LC3 linker compounds by SMM-based screening and validation.

c-e) For all association-dissociation curves, vertical dash lines mark the starts of association and dissociation phases of the binding event. The red dash curves are global fits to a Langmuir reaction model with the fitting parameters listed at the bottom of each plot. No binding signals were observed for HTTexon1-Q25-MBP or full-length HTT-Q23 proteins, and thus the parameters were not presented.

Figure 4. Linker compounds enhanced mHTT-LC3 interaction and tethered $\mathrm{mHTT}$ to autophagosomes.

a-b) For MBP pull-down (a), purified HTTexon1-MBP $(10 \mu \mathrm{g})$ or MBP $(10 \mu \mathrm{g})$ bound MBP-resin were incubated with the purified LC3B protein $(40 \mu \mathrm{g})$ and the indicated compounds. The HTTexon1-MBP or the MBP proteins were pulled down and the eluates were tested for co-precipitated LC3B. $4 \%$ of the total eluate was loaded in each lane, and the input : pull-down loading ratio was 100\%. Both 1005 and AN2 enhanced LC3B's interaction with HTTexon1-Q72-MBP, but not HTTexon1-Q25-MBP. Note that the MBP blot signals were much weaker for the Q72 protein, possibly because recognition of the MBP tag by the antibody was affected in the fusion protein. Meanwhile, data interpretation was not influenced, because compound treatments did not alter the MBP signals for the Q72 protein (last three lanes). The GST pull-down (b) was performed similarly, except using full-length HTT-Q73 or full-length HTT-Q23 (both without fusion tags) and GST-LC3B proteins for the in vitro GST pull-down experiments to precipitate GST-LC3B or GST alone with its binding proteins, and then eluted for detection. Note that the pull-down is in the reverse direction of the pull-down in (a). The input : pull-down loading ratio for the GST blot was $100 \%$, whereas the ratio for the HTT blot was $10 \%$ to avoid over-exposure of the input. Both 1005 and AN2 enhanced LC3B's interaction with the full-length HTT-Q73 but not the full-length HTT-Q23 protein.

c-d) Representative confocal microscopy images (scale bar: $10 \mu \mathrm{m}$ ) and quantifications of the co-localization between HTTexon1-MBP-His (red, detected by anti-His immunofluorescence) and LC3B-GFP (green, detected by GFP fluorescence directly) in transiently transfected HeLa cells (c) or between endogenous mHTT and

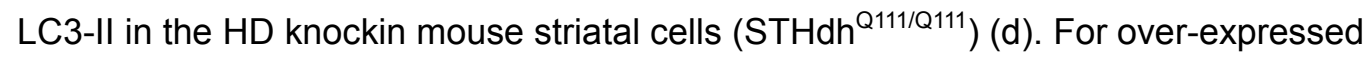
proteins (c), the LC3B-GFP alone transfected or the HTTexon1-MBP-His alone transfected cells were imaged at both channels to ensure the specificity of the signals (upper panels). The white arrows indicate representative co-localization puncta. Parts 
of the images have been amplified to show co-localization puncta more clearly (pointed by orange arrows). Since the puncta were obvious, co-localization was analyzed by counting the red $^{+}$green ${ }^{+}$(yellow) and the total red $^{+}$puncta directly, and then calculate their ratio for each cell. Blind analysis was performed for quantifications. For endogenous proteins (d), mHTT was detected by the anti-HTT antibody 2166 , and the endogenous LC3-II was detected by an anti-LC3 antibody that has been reported to specifically detect LC3-II ${ }^{32}$. Since the signals of endogenous proteins were more dispersed, the co-localization analysis was performed blindly by measuring the red $^{+}$green ${ }^{+}$(yellow) and the total red ${ }^{+}$pixels by ImageJ, and then calculate their ratio for each cell.

Figure 5. Linker compounds rescued HD-relevant phenotypes in cells and in vivo. a) Loss of processes and shrinkage of neurons were observed in HD neurons after BDNF removal. Bar plots: quantification of the TUBB3 signal covered area (TUBB3 area) normalized to the nuclei counts based on DAPI. The lower TUBB3 area per cell reflects neuronal processes shrinkage and loss. Data were normalized to the average of WT controls. The statistical analysis was performed by one-way $\operatorname{ANOVA}(F(5,60)=$ 94.78) with post-hoc Dunnett's tests: “****”: $p<0.0001$. The n number indicates the number of independently plated wells.

b) The images were captured every 3 hours inside the incubator using Incucyte, and the caspase- 3 active cells were quantified by the fluorescent object count per field. The statistical analysis was performed by two-way $\operatorname{ANOVA}(F(43,516)=12.85)$ with post-hoc Dunnett's tests, comparing to the HD_DMSO group. “****”: $p<0.0001$. The numbers in brackets indicate the number of independently plated wells, with 4 fields per well imaged and averaged for quantification. Three batches were tested and showed consistent results.

c) Left: the Drosophila expressed full-length HTT proteins (Q128 or Q16) in the nervous system driven by elav-GAL4. 75 flies were tested for each group. The statistics was performed by Log-rank (Mantel-Cox) test, comparing compound treated groups with DMSO controls in Q128 flies. ${ }^{* * * *}: p<0.0001$. Right: similar as the left panel, but plotting the climbing performance as a function of age after eclosion. The statistics was performed by two-way ANOVA $(F(4,275)=122.1)$ with post-hoc Dunnett's tests, comparing the compound treated groups with the DMSO controls in Q128 flies. Numbers in brackets indicate the number of vails (each containing 15 flies) tested. ${ }^{* * * *}: p<0.0001$. d-f) The numbers in brackets indicated the number of mice tested. The statistical analysis was performed by two-way ANOVA with post-hoc Dunnett's tests, and the $p$ 
559

560

561

562

563

564

565

566

567

568

569

570

571

572

573

574

575

576

577

578

579

580

581

582

583

584

585

586

587

588

589

590

591

592

593

594

values compared with the DMSO control were indicated (“****”: $p<0.0001$ ). For HD mice, $F(2,195)=4.963$ in rotarod tests, $F(2,195)=37.31$ in balance beams tests, and $F(2,156)=7.068$ in gripping force tests. No significant difference was detected in the wild-type mice injected with different compounds. The investigators were blinded to the compounds and genotypes when performing the experiments. In all panels, the error bars represent mean and s.e.m..

\section{Compound stamping on the microarray}

Small molecule microarrays (SMMs) containing 3,375 bioactive compounds were used for high-throughput screening of target proteins. The compound library containing 1,527 drugs approved by Food and Drug Administration (FDA) of United States, 1,053 natural products from traditional Chinese medicine, and 795 known inhibitors were stamped onto the SMMs. Each compound was dissolved in DMSO at a concentration of $10 \mathrm{mM}$ and printed in duplicates along vertical direction on homemade phenyl-isocyanate functionalized glass slides with a contact microarray printer (SmartArrayer 136, CapitalBio Corporation). Biotin-BSA at a concentration of $7,600 \mathrm{nM}$ in $1 \times$ phosphate-buffered saline (PBS) and biotin-(PEG) $)_{2}-\mathrm{NH}_{2}$ at a concentration of $5 \mathrm{mM}$ in DMSO were printed as the inner and outer borders of SMMs, respectively. The diameter of each spot was about $150 \mu \mathrm{m}$ and spacing between two adjacent spots was $250 \mu \mathrm{m}$. The printed SMMs were then dried at $45^{\circ} \mathrm{C}$ for $24 \mathrm{~h}$ to facilitate covalent bonding of nucleophilic groups of small molecules to isocyanate groups of the functionalized slides. Afterwards, the SMMs were stored in a $-20{ }^{\circ} \mathrm{C}$ freezer.

\section{Expression and purification of recombinant proteins}

The human microtubule associated protein 1 light chain 3 beta (MAP1LC3B, LC3B) gene (GenBank: NM_022818.4) was amplified by PCR and cloned into a pGEX-6P1 (GE Healthcare) derived vector pGHT, which is a prokaryotic expression vector reconstructed by adding a His8 tag and a TEV protease cleavage site before the pGEX-6P1 multiple cloning site. After sequencing verification, the expression plasmid pGHT-LC3B was introduced into Escherichia coli BL21 (DE3) pLsyS, in which the recombinant GST-LC3B protein was expressed by IPTG induction. When the bacterial culture reached $\mathrm{OD}_{600}=0.8$, its temperature was decreased to $18{ }^{\circ} \mathrm{C}$, and 0.2 mM IPTG was added into the culture for an additional $20 \mathrm{~h}$ incubation. The cells were then harvested by centrifugation $\left(6,000 \mathrm{~g}, 4{ }^{\circ} \mathrm{C}, 15 \mathrm{~min}\right)$ and the cell pellet was suspended in $50 \mathrm{mM}$ Tris- $\mathrm{HCl}$ buffer, $\mathrm{pH} 7.5$, with $150 \mathrm{mM} \mathrm{NaCl}$ and $5 \%$ glycerol. Cells were then disrupted by sonication, followed by centrifugation $\left(20,000 \mathrm{~g}, 4^{\circ} \mathrm{C}, 60\right.$ min). The supernatants were then loaded onto a HisTrap HP column (GE Healthcare, 
cat. no. 17524701), and eluted with $50 \mathrm{mM}$ Tris-HCl buffer, $\mathrm{pH}$ 7.5, containing $150 \mathrm{mM}$ $\mathrm{NaCl}, 5 \%$ glycerol and $300 \mathrm{mM}$ imidazole. The LC3B eluate was then mixed with TEV protease (Sigma, cat. no. T4455; eluted protein: TEV protease $=100: 1$ ) and dialyzed against the dialysate buffer (50 mM Tris- $\mathrm{HCl}$ buffer, $\mathrm{pH} 7.5$, containing $100 \mathrm{mM} \mathrm{NaCl}$ ) in $4{ }^{\circ} \mathrm{C}$ overnight. After TEV protease treatment, the samples were then loaded onto a HisTrap HP column again, the flow through fraction which mainly contains tag removed recombinant LC3B. Afterwards, the proteins were concentrated and further purified by Superose 6 Increase 10/300 GL (GE Healthcare) size exclusive chromatography. Finally, the purified proteins were concentrated to approximately 10 $\mathrm{mg} / \mathrm{ml}$ in $50 \mathrm{mM}$ HEPES buffer with $100 \mathrm{mM} \mathrm{NaCl}$ for further analysis. The MBP-His8 and Rpn10 proteins were purified similarly.

The full-length HTT proteins, HTTexon1-MBP, polyQ-sfGFP and sfGFP proteins were purified from mammalian cells. For full-length HTT proteins, the human HTT gene (GenBank: NM_002111.8) with (CAG) $)_{23}$ or $(C A G)_{73}(23 Q$ or $73 Q$ for proteins) were de novo synthesized (by Genewiz Inc.), sequence validated and then cloned into a modified $\mathrm{pCAG}$ vector with an $\mathrm{N}$-terminal protein $\mathrm{A}$ tag. The plasmid was transfected to human embryonic kidney E293 cells using polyethylenimine (PEI, from Polysciences, cat. no. 23966). After culture at $37{ }^{\circ} \mathrm{C}$ for 48 to $60 \mathrm{~h}$, cells were collected and lysed at $4{ }^{\circ} \mathrm{C}$ for $1 \mathrm{~h}$ in lysis buffer containing $50 \mathrm{mM}$ Tris- $\mathrm{HCl}, \mathrm{pH}$ 8.0, $150 \mathrm{mM} \mathrm{NaCl}, 5 \%$ glycerol, 0.5\% CHAPS, $3 \mathrm{mM} \mathrm{DTT}, 1 \%$ PMSF, $1 \mu \mathrm{g} / \mathrm{ml}$ pepstatin, 1 $\mu \mathrm{g} / \mathrm{ml}$ leupeptin and $1 \mu \mathrm{g} / \mathrm{ml}$ aprotinin, $5 \mathrm{mM}$ ATP and $5 \mathrm{mM} \mathrm{MgCl}$. After centrifugation at $15000 \mathrm{rpm}$ for $40 \mathrm{~min}$, the supernatants were then incubated with IgG monoclonal antibody-agarose (Smart-lifesciences, cat. no. SA030010) for $2 \mathrm{~h}$ and unbound proteins were extensively washed away. The HTT proteins were then digested using TEV protease overnight to remove the protein $A$ tag and eluted protein was further purified by ion exchange and gel filtration chromatography using Mono $Q$ and Superose $6(5 / 150 \mathrm{GL})$ columns from GE healthcare. The peak fractions were pooled for further biochemical analysis. The HTTexon1 with 25Q or 72Q cDNA were also de novo synthesized and cloned into a mammalian expression vector pTT5SH8Q2 for large scale production in HEK293T cells. In order to improve the production yield and increase the solubility, a $C$ terminal MBP tag was added after the HTTexon1 sequences to generate the pTT-HTTexon125Q-MBP and PTT-HTTexon125Q-MBP plasmids. For protein production and purification, the HEK293T cells were transfected by PTT-HTT25QExon1-MBP and pTT-HTT72QExon1-MBP plasmids with linear PEI (PolySciences \#24765), and then collected after $48 \mathrm{~h}$. The cells were then lysed by sonication in buffer containing 50 $\mathrm{mM}$ Tris- $\mathrm{HCl}, \mathrm{pH}$ 7.5, $150 \mathrm{mM} \mathrm{NaCl}, 20 \mathrm{mM}$ imidazole, 5\% glycerol, protease inhibitor 
632

633

634

635

636

637

638

639

640

641

642

643

644

645

646

647

648

649

650

651

652

653

654

655

656

657

658

659

660

661

662

663

664

665

666

667

cocktail (Sigma) and $50 \mathrm{U} / \mathrm{ml}$ benzonase (Sigma). After centrifugation, the supernatants were loaded onto HisTrap HP column (GE Healthcare), and eluted with the buffer containing $50 \mathrm{mM}$ Tris- $\mathrm{HCl}, \mathrm{pH} 7.5,150 \mathrm{mM} \mathrm{NaCl}, 300 \mathrm{mM}$ imidazole, 5\% glycerol and protease inhibitor cocktail. The MBP tag was not cleaved to avoid precipitation. Afterwards, the proteins were concentrated and further purified by Superose 6 Increase 10/300 GL (GE Healthcare) size exclusive chromatography.

Verifications of the recombinant proteins by matrix assisted laser desorption ionization-time of flight mass spectrometry (MALDI-TOF).

The purified LC3B, HTTexon1Q25-MBP, and HTTexon1Q72-MBP proteins were dialyzed into $5 \mathrm{mM} \mathrm{NH}_{4} \mathrm{Ac}$ by Superose 6 Increase size exclusive chromatography for linear mode MALDI-TOF analysis on a Bruker FLEX MALDI-TOF instrument. A total of 1500-2500 scans were averaged for each spectrum using an accelerating voltage of $25 \mathrm{kV}$. Sinapinic acid (SA, Bruker, cat. no. 820135) was used as the matrices for protein and peptide analyses. SA was made into $20 \mathrm{mg} / \mathrm{mL}$ solutions in $70 \% \mathrm{ACN}, 0.1 \%$ TFA. For the acquisition of spectra from 10,000 to $100,000 \mathrm{amu}, 2 \mu \mathrm{L}$ of sample was mixed with $2 \mu \mathrm{L}$ of SA solution in an Eppendorf tube, and $2 \mu \mathrm{L}$ of the mixture was loaded onto the MALDI plate. The calibration peptides for this range were BSA ( $M+$ 66431) (Sigma, cat. no. A1933). All spectra were obtained in positive linear mode. The amount of full-length HTT proteins were limited, and thus not validated by MALDI-TOF. Instead, they were further purified by ion exchange and gel filtration chromatography, and validated by Coomassie-blue staining (Extended data Fig. 1e) and Western-blots (Fig. 4b).

\section{Verifications of the recombinant LC3B by $X$ ray diffraction crystallography}

Since the deletion of G120 (lipidation site) stabilizes LC3B protein, we utilized LC3B $\Delta$ G120 protein to get high resolution diffraction data. Purified LC3B $\Delta$ G120 protein was concentrated in the following buffer: $20 \mathrm{mM} \mathrm{HEPES} \mathrm{pH7.5,} 150 \mathrm{mM} \mathrm{NaCl}$. The LC3B $\Delta$ G120 crystal was grown in reservoir solutions consisting of $0.16 \mathrm{M}$ ammonium sulfate, $0.08 \mathrm{M}$ sodium acetate $\mathrm{pH}$ 4.6, 20\% (w/v) PEG4000, 20\% (v/v) glycerol and $0.01 \mathrm{M}$ Taurine.

\section{Refinement}

The X-ray diffraction data were collected at $100 \mathrm{~K}$ in the beamline BL17U1 and BL19U1, SSRF. The wavelength for data collection was $0.97892 \AA$. Diffraction images were indexed and processed by HKL2000. The structure of LC3B $\triangle$ G120 (PDB ID $6 J 04,1.90 \AA$ ) was solved by molecular replacement with the Phaser 2.8 program from the CCP4 crystallography package using the (PDB ID code 1UGM) as the search model. The refinement was performed by Refmac 5.5 and Phenix 1.14. There are no 
668 Ramachandran outliers to report. The related figure was drawn using PyMOL 2.2.

669 Compound-protein interaction measurements by oblique-incidence reflectivity 670 difference (OI-RD)

671 For high-throughput preliminary screening of target proteins, a SMM was 672 assembled into a fluidic cartridge and washed in situ with a flow of $1 \times$ PBS to remove 673 excess unbound small molecules. After washing, the SMM was scanned with a 674 label-free OI-RD scanning microscope to image small molecules immobilized on glass 675 slides. After it was blocked with 7,600 nM BSA in 1× PBS for 30 min, SMM was 676 incubated with the target protein for $2 \mathrm{~h}$. HTTexon1-Q25-MBP at a concentration of $677454 \mathrm{nM}$, HTTexon1-Q72-MBP at a concentration of $238 \mathrm{nM}$, and LC3B at a 678 concentration of $680 \mathrm{nM}$ were screened on separate fresh SMMs. OI-RD images were 679 scanned for each operation, including washing, blocking, and incubation. The OI-RD 680 difference images (images after incubation - images before incubation) were utilized 681 for analysis, and vertical bright doublet spots indicated compounds that bind with 682 target proteins in both replicates. $8 \mathrm{~F} 20$ and 1005 were identified to bind to 683 HTTexon1-Q72-MBP and LC3B, but not HTTexon1-Q25-MBP. The binding was 684 further confirmed by the kinetics measurements (see below).

685 To measure binding kinetics of target proteins with compounds, we prepared new 686 SMMs consisting of 8F20,1005, and AN2. Six identical microarrays were printed on 687 one glass slide and each compound was printed in triplicates in a single microarray. 688 The printed small SMMs were assembled into a fluidic cartridge with each microarray 689 housed in a separate chamber. Before the binding reaction, the slide was washed in 690 situ with a flow of $1 \times$ PBS to remove excess unbound samples, followed by blocking 691 with 7,600 nM BSA in $1 \times$ PBS for 30 min. For binding kinetics measurement, $1 \times$ PBS 692 was first flowed through a reaction chamber at a flow rate of $0.01 \mathrm{~mL} / \mathrm{min}$ for $5 \mathrm{~min}$ to 693 acquire the baseline. $1 \times$ PBS was then quickly replaced with the probe solution of the 694 target protein at a flow rate of $2 \mathrm{~mL} / \mathrm{min}$ for $9 \mathrm{sec}$ followed by a reduced flow rate at $6950.01 \mathrm{~mL} / \mathrm{min}$ to have the microarray incubated in the probe solution under the flow 696 condition for $35 \mathrm{~min}$ (association phase of the reaction). The probe solution was then 697 quickly replaced with $1 \times$ PBS at a flow rate of $2 \mathrm{~mL} / \mathrm{min}$ for $9 \mathrm{sec}$ followed by a 698 reduced flow rate of $0.01 \mathrm{~mL} / \mathrm{min}$ to allow dissociation of probe for $30 \mathrm{~min}$ 699 (dissociation phase of the reaction). By repeating the binding reactions of the target 700 protein at three different concentrations on separate fresh microarrays, binding curves 701 of compounds with the target protein at three concentrations were recorded with 702 scanning OI-RD microscope. Reaction kinetic rate constants were extracted by fitting 703 the binding curves globally using 1-to-1 Langmuir reaction mode.

704 Compound-protein interaction measurements by microscale thermophoresis 
The purified recombinant proteins were dialyzed into $1 \times$ PBS, and then labelled according to the protocol of Protein labeling kit RED-NHS (Nanotemper, cat. no. L001). All the tested stock compounds $(10 \mathrm{mM})$ dissolved in DMSO were also diluted into the same buffer for the final MST assay. The MST experiment was performed using Monolith NT.115 instrument (NanoTemper Technologies). $500 \mathrm{nM}$ of labelled proteins were mixed with the indicated concentrations of candidate compounds in the reaction buffer containing $20 \mathrm{mM}$ HEPES, pH 7.4, $150 \mathrm{mM} \mathrm{NaCl}$. The MST data were then collected under $40 \%$ infrared laser power and $20 \%$ light-emitting diode power. The data were analyzed by Nanotemper analysis software (1.5.41) and the $K_{d}$ was determined.

\section{cDNA plasmids for transfection in mammalian cells}

The pEX-GFP-hLC3WT plasmid was obtained from Addgene (\#24987) to express LC3B. The pTT-HTTexon1-Q72-MBP-His and pTT-HTTexon1-Q25-MBP-His were generated by subcloning HTTexon 1 cDNAs into the mammalian expression vector pTT-MBP-His and then transiently transfected into HeLa cells to express HTTexon1 proteins for the colocalization experiments. The polyQ-GFP sequences (expressing Met-polyQ-sfGFP) were de novo synthesized and subcloned into the pcDNA vector. All plasmids were sequence validated. For transient transfections, the cells were plated at $50 \%$ confluence. After $24 \mathrm{~h}$, the cDNAs were transfected with Lipofectamine 2000 (ThermoFisher Scientific, cat. no. 11668019) using the forward transfection protocol provided by the manufacturer.

\section{Cell culture}

For mouse primary cortical neuron cultures, cortices were isolated from P0 pups following genotyping. Cortices were dissected into cold $\mathrm{Ca}^{2+}$ - and $\mathrm{Mg}^{2+}$-free PBS buffer. Chopped small pieces were digested in solution containing $2.5 \%$ trypsin (Sigma, cat. no. P1005) and DNase I (0.1 mg/mL, Sigma, cat. no. D5025), for 20-30 $\min$ at $37^{\circ} \mathrm{C}$. Tissues were transferred to $10 \%$ FBS containing DMEM (ThermoFisher Scientific, cat. no. 11965) to cease digestion. Neurons were then dissociated by trituration with fire-polished glass pipettes, collected by spinning and plated onto polylysine-coated dishes at $4 \times 10^{5}$ cell $/ 35 \mathrm{~mm}$ dish. The growth medium was composed of Neurobasal A medium (ThermoFisher Scientific, cat. no. 10888022) with 1× B-27 (ThermoFisher Scientific, cat. no. 17504044) and 1× N2 supplement (ThermoFisher Scientific, cat. no. 17504048). Cytosine-arabinofuranoside (Sigma, cat. no. C1768) was added at $6 \mu \mathrm{M}$ to inhibit glial growth.

Some of the primary patient fibroblasts were obtained from HD patients (Q47, Q49, Q55) and healthy sibling (WT, Q19) controls in a Mongolian Huntington's 
disease family. The HD Q68 fibroblast line was obtained from Coriell Cell Repositories (Camden, NJ, USA). The PD line was obtained from an idiopathic Parkinson's disease patient, and the SCA3 line was obtained from a SCA3 patient with the ATXN3 expansion mutation (Q74). The studies were approved by The Ethic Community of Institutes of Biomedical Sciences at Fudan University (\#28) for obtaining the HD and wild-type patient fibroblasts, and by Huashan Hospital Institutional Review Board at Fudan University (\#174) for obtaining the PD and SCA3 patient fibroblasts. Verbal and written consent was obtained from patients. The procedures were in compliance with all relevant ethical regulations. The immortalized fibroblasts were generated by infection of lentivirus expressing SV40T. For generation of iPS cells (iPSCs), the primary fibroblasts were transduced with the retroviral STEMCCA polycistronic reprogramming system (Millipore, cat. no. SCR548). The iPSCs were confirmed positive for Tra-1-81, Tra-1-60, SSEA-4 and Nanog by immunofluorescence and flow-cytometry. All four vector-encoded transgenes were found to be silenced and the karyotype was normal. iPSCs were cultured in E8 medium (ThermoFisher Scientific, cat. no. A1517001) on Matrigel (Corning, cat. no. 354277) surface. iPSCs were differentiated to Pax6-expressing primitive neuroepithelia (NE) for 10-12 days in a neural induction medium. Sonic hedgehog $(\mathrm{SHH}, 200 \mathrm{ng} / \mathrm{ml})$ was added at days 10-25 to induce ventral progenitors. For neuronal differentiation, neural progenitor clusters were dissociated and placed onto poly-ornithine/laminin-coated coverslips at day 26 in Neurobasal medium (ThermoFisher Scientific, cat. no. 21103049), with 1x B-27 (ThermoFisher Scientific, cat. no. 17504044), 1× N-2 (ThermoFisher Scientific, cat. no. 17504048), brain derived neurotrophic factor (BDNF, $20 \mathrm{ng} / \mathrm{ml}$, Protech, cat. no. 450-02), glial-derived neurotrophic factor (GDNF, $10 \mathrm{ng} / \mathrm{ml}$, Protech, cat. no. 450-10), insulin-like growth factor 1 (IGF1, $10 \mathrm{ng} / \mathrm{ml}$, Protech, cat. no. 100-11) and Vitamin C (Sigma cat. no. D-0260, $200 \mathrm{ng} / \mathrm{ml}$ ). The mouse striatal cells (STHdh) were obtained from Coriell Cell Repositories (Camden, NJ, USA). The HEK293T cells and the HeLa cells were originally obtained from American Type Culture Collection (ATCC). STHdh, HeLa and HEK293T cells were cultured in DMEM (ThermoFisher Scientific, cat. no. 11965) with 10\% (vol/vol) FBS (ThermoFisher Scientific, cat. no. 10082-147). Atg5 WT and KO MEFs were from N. Mizushima. All the mammalian cell lines were maintained at $37{ }^{\circ} \mathrm{C}$ incubator with $5 \% \mathrm{CO}_{2}$, except STHdh cells, which were maintained at $33{ }^{\circ} \mathrm{C}$ with $5 \% \mathrm{CO}_{2}$. The cells were tested every two months by a TransDetect PCR Mycroplasma Detection Kit (Transgen Biotech, cat. no. FM311-01) to ensure that they are mycoplasma free. The CellTiter-glo assay was performed to measure cell viability with the indicated compound treatment (Extended Data Fig. 2e) following the protocol provided in the kit (Promega, cat. no. G7570). 
779

780

781

782

783

784

785

786

787

788

789

790

791

792

793

794

795

796

797

798

799

800

801

802

803

804

805

806

807

808

809

810

811

812

813

814

\section{HD Drosophila models}

The nervous system driver line elav-GAL4 (c155), and the HTT-expressing lines UAS-fIHTT-Q16 and the UAS-fIHTT-Q128 (expressing human full-length HTT with $16 Q$ and $128 Q$, respectively, when crossed to the GAL4 line) lines were obtained from the Bloomington Drosophila Stock Center at University of Indiana (http://flystocks.bio.indiana.edu/), and maintained in a $25^{\circ} \mathrm{C}$ incubator. Crosses were set up between virgin female flies carrying elav-GAL4 driver and the UAS-fIHTT-Q16 or UAS-fIHTT-Q128 male flies to generate the desired genotypes.

\section{HD mouse models}

The generation and characterization of the Hdh140Q knock-in mice have been previously described ${ }^{19}$. Mice were group-housed (up to 5 adult mice per cage) in individually vented cages with a $12 \mathrm{~h}$ light/dark cycle. The mouse experiments were carried out following the ARRIVE (Animal Research: Reporting of In Vivo Experiments) guidelines, and they were in compliance with all relevant ethical regulations. The Animal Care and Use Committee of the School of Medicine at Fudan University approved the protocol used in animal experiments (Approval \#20140904 and \#20170223-005).

\section{Compound treatment in cells and animals}

The compounds utilized in this study were all commercially available, and quality controlled by the vendors using NMR. 1005: GW5074 (DC Chemicals; cat.no. DC8810); 8F20: ispinesib (Selleck; cat.no. S1452); AN1: 5-bromo-3-[(4-hydroxyphenyl)methylidene]-2,3-dihydro-1H-indol-2-one (Specs; cat. no. AN-655/15003575); AN2: 5,7-Dihydroxy-4-phenylcoumarin (ChemDiv; cat.no. D715-2435); GSK923295 (Selleck, cat.no. S7090), BAY1217389 (Selleck, cat.no. S8215), PLX-4720 (Selleck, cat.no. S1152), Dabrafenib (Selleck, cat.no. S2807), Sorafenib Tosylate (Selleck, cat.no. S1040), rapamycin (Sigma-Aldrich, cat. no. R8781).

For compound treatment in the cells, the compounds were diluted in culture medium to $10 \mathrm{X}$ concentrations and added to the plated cells: for primary cultured neurons and iPSC-derived neurons, the compounds were added 5 days after plating; for patient fibroblasts and other cell lines, the compounds were added 1 day after plating. The cells were then collected 2 days later for measurement of HTT levels. For detection of HTT-LC3 colocalization, the cells were fixed $4 \mathrm{~h}$ after compound treatment. For caspase-3 activation detection, the cells were stressed (BDNF removal for iPSC-derived neurons) 1 day after compound treatment, and tested at the indicated time points. 
For compound treatment in the Drosophila, flies were maintained in standard maize food at $25^{\circ} \mathrm{C}$. For drug feeding, maize media was heated to $45^{\circ} \mathrm{C}$ until liquid and distributed into vials. Compounds were freshly prepared in DMSO and added to the media. New adult flies were transferred to vials with $400 \mu \mathrm{L}$ the control (DMSO) or compound-containing food, which was changed every other day.

For compound treatment in mice using intracerebral ventricular (icv) injection, the 3-month-old mice were anesthetized using a small animal anesthesia machine (MSS-3, MSS International, Keighley, UK) by Isoflurane (1.5\% solution). We surgically implanted each mouse with a guide cannula directed toward the lateral ventricle. The coordinates for implantation were determined utilizing "The Mouse Brain in Stereotaxic Coordinates" and the guide cannulas were placed at $0.6 \mathrm{~mm}$ posterior, $1.5 \mathrm{~mm}$ lateral (left), and $1.7 \mathrm{~mm}$ dorsal with respect to bregma. A cap with stylus was then inserted into the guide cannula to seal its opening. Mice were then allowed to recover from surgery for a week before being treated. For injection, we first inserted an internal injector cannula so that it extended $0.5 \mathrm{~mm}$ beyond the tip of the guide cannula to reach the lateral ventricle. We then injected the mice through the internal injector cannula using a $25 \mu \mathrm{L}$ syringe (Hamilton 1700 Series Microliter Syringes , Bonaduz, $\mathrm{GR}, \mathrm{CH}$ ) at a flow rate of $0.25 \mu \mathrm{L} / \mathrm{min}$ powered by a syringe pump (KDS Legato 130, Holliston, MA, USA) to administer $2 \mu \mathrm{L}$ of compounds-containing artificial cerebrospinal fluid (ACSF: $1 \mathrm{mM}$ glucose, $119 \mathrm{mM} \mathrm{NaCl}, 2.5 \mathrm{mM} \mathrm{KCl}, 1.3 \mathrm{mM} \mathrm{MgSO}_{4}$, $2.5 \mathrm{mM} \mathrm{CaCl}_{2}, 26.2 \mathrm{mM} \mathrm{NaHCO}, 1 \mathrm{mM} \mathrm{NaH} \mathrm{PO}_{4}$ ) at a concentration of $25 \mu \mathrm{M}$ (containing $0.125 \% \mathrm{vol} / \mathrm{vol} \mathrm{DMSO}$ ). $2 \mu \mathrm{L}$ ACSF containing equivalent amount of DMSO $(0.125 \% \mathrm{vol} / \mathrm{vol})$ was used as the control. The injector cannula was left in place for approximately $60 \mathrm{~s}$ to allow for diffusion before placing the caps with stylus back in guide cannulas.

For compound treatment in mice using intraperitoneal (ip) injection, each mouse was weighed. The compounds were diluted with $0.9 \% \mathrm{NaCl}$ intravenous infusion solution to $0.05 \mu \mathrm{g} / \mu \mathrm{L}$ (containing $0.011 \mu \mathrm{g} / \mu \mathrm{L} \mathrm{DMSO}$ ) and injected into each mouse based on the weight of the mouse $(500 \mu \mathrm{g} / \mathrm{kg}$, containing $110 \mu \mathrm{g} / \mathrm{kg} \mathrm{DMSO})$. As controls, equivalent amount of DMSO was diluted and injected in the same way. Injection of $0.9 \% \mathrm{NaCl}$ intravenous infusion solution alone was also tested and showed no difference (Extended data Fig. 9f-h). One injection per day was performed for two weeks before subsequent behavioral experiments or tissue extractions.

Note that in some of the experiments (Fig. 4-5 \& Extended Data Fig. 6b-d), 8F20 and/or AN1 were not tested. 8F20 was not tested because it did not have an effect in vivo by icv-injection (Extended Data Fig. 6a). AN1 was not tested because its 
851 structure is highly similar as 1005 while it had a weaker HTT-lowering effect by

852 icv-injection (Extended Data Fig. 6a).

\section{Protein extraction from cells and tissues}

854 For protein extraction from cells, the cell pellets were collected and lysed on ice 855 for $30 \mathrm{~min}$ in $1 \times \mathrm{PBS}+1 \%$ Triton X-100+1 $\times$ complete protease inhibitor (Sigma-Aldrich, 856 cat. no. 11697498001 ), sonicated for $10 \mathrm{sec}$, and spun at $>20,000 \mathrm{~g}$ at $4{ }^{\circ} \mathrm{C}$ for $15 \mathrm{~min}$. 857 The supernatants were then loaded and transferred onto nitrocellulose membranes 858 for Western-blots. For mouse brain tissues, the mouse striata and cortices were 859 dissected on ice and grinded by a tissue grinder for $5 \mathrm{~min} 60 \mathrm{~Hz}$ and lysed on ice for $86060 \mathrm{~min}$ in brain lysis buffer (50 mM Tris, $250 \mathrm{mM} \mathrm{NaCl}, 5 \mathrm{mM}$ EDTA, 1\% Triton X-100 $861 \mathrm{PH} 7.4)+1 \times$ complete protease inhibitor (Roche, cat. no. 4693159001). The samples 862 were then sonicated for 10 cycles, $15 \mathrm{~s}$ on and $20 \mathrm{~s}$ off, and then collected for 863 Western-blots.

864 For protein extraction from the mouse brain, the brains were collected and the 865 cortices were acutely dissected on ice and homogenized with a tissue grinder for 5 $866 \mathrm{~min}$ at $60 \mathrm{~Hz}$ and lysed on ice for $60 \mathrm{~min}$ in brain lysis buffer (50 mM Tris, $250 \mathrm{mM}$ $867 \mathrm{NaCl}, 5 \mathrm{mM}$ EDTA, 1\% (vol/vol) Triton X-100, 1× complete protease inhibitor (Roche, 868 cat. no. 4693159001$), \mathrm{pH}=7.4)$. The samples were then sonicated for 10 cycles, $15 \mathrm{~s}$ 869 on and $20 \mathrm{~s}$ off, and then collected for Western-blots, HTRF or dot blots.

870 For mHTT measurements in the HD Drosophila model, the fly heads were 871 collected at the age of 7 days and lysed on ice for $30 \mathrm{~min}$ in PBS $+1 \%$ (vol/vol) Triton 872 X-100 + 1× complete protease inhibitor (Roche, cat. no. 4693159001), sonicated for 87310 cycles, $15 \mathrm{~s}$ on and $20 \mathrm{~s}$ off, and then collected for HTRF.

874 For all the samples, the protein concentrations were measured to correct the 875 loadings. Different protein concentrations or cell numbers per well were tested to 876 ensure that the signals were in the linear range. Background corrections were 877 performed by subtracting the background signals from blank samples.

\section{Western-blot and filter trap assays}

879 For Western-blots, the samples were loaded onto the SDS page gel $(5-12 \%$ 880 depending on the molecular weight of the protein of interest). The proteins on the gel 881 were then transferred to the nitrocellulose membranes for blocking and antibody 882 detection. The signal was detected with ECL (Bio-Rad, cat. no. 1705061) after $1 \mathrm{~h}$ 883 incubation of the membrane with secondary antibody $1: 10,000$.

884 The filter trap assay was performed similarly as previously described ${ }^{23}, 2 \mu \mathrm{L}(10$ $885 \mu \mathrm{g}$ ) aliquots of each sample were loaded onto nitrocellulose membranes stacked in 886 the Bio-Dot microfiltration apparatus (Bio-Rad). The membrane was blocked for $1 \mathrm{~h}$ 887 with 5\% milk and incubated overnight with the antibody 4C9 at a concentration of 1.5 
$888 \mu \mathrm{g} / \mu \mathrm{l}$ in $5 \%$ milk diluted in PBS $+0.1 \%$ Tween-20. The signal was detected with ECL

889 (Bio-Rad, cat. no. 1705061) after $1 \mathrm{~h}$ incubation of the membrane with secondary

890 antibody 1:10,000.

891 Homogeneous Time Resolved Fluorescence (HTRF) assays

892 For HTRF, the assays were similar as previously described ${ }^{25}$. The cell or tissue 893 lysates were diluted with the original lysis buffer PBS $+1 \%$ (vol/vol) Triton X-100 + 1x 894 complete protease inhibitor (Roche), utilized for lysing the samples, and then detected 895 with indicated antibody pairs diluted in the HTRF assay buffer (50 mM NaH ${ }_{2} \mathrm{PO}_{4}, 400$ $896 \mathrm{mM} \mathrm{NaF}, 0.1 \%$ BSA, 0.05\% (vol/vol) Tween-20, 1\% (vol/vol) Triton X-100, pH 7.4). 897 The donor antibody concentration was $0.023 \mathrm{ng} / \mu \mathrm{L}$ and the acceptor antibody 898 concentration was $1.4 \mathrm{ng} / \mu \mathrm{L}$, both in HTRF assay buffer. Different antibody pairs were 899 used for different experiments as indicated in the figure legends. For all the samples, 900 the signals were normalized to the total protein concentrations to ensure equal 901 loadings. Different protein concentrations were pre-tested to ensure that the signals 902 were in the linear range. Background corrections were performed by subtracting the 903 background signals from blank samples.

904 In vitro c-Raf kinase assay

905

In vitro c-Raf kinase assays were carried out with a c-Raf kinase assay kit (BPS 906 Bioscience, cat. no. 79570). The assays were performed in a 96-well plate according 907 to the manufacturing instruction. The samples and non-reactive negative controls 908 were tested in duplicate according to the instruction.

909 For details, $25 \mu \mathrm{L}$ of the mixture containing $5 x$ kinase assay buffer $(6 \mu \mathrm{L})$, ATP $(1 \mu \mathrm{L})$, $9105 x$ Raf substrate $(10 \mu \mathrm{L})$ and water $(8 \mu \mathrm{L})$ was added to a well. $5 \mu \mathrm{L}$ of water solution 911 containing a test compound at a 10x desired concentration (DMSO was at $10 \%$ at the 912 water solution) was added to the $25 \mu \mathrm{L}$ of mixture, and $20 \mu \mathrm{L}$ of $1 \mathrm{x}$ kinase assay 913 buffer containing $2 \mathrm{ng} / \mu \mathrm{L} \mathrm{c}$-Raf kinase was added to the mixture in a well to initiate the 914 kinase reaction (at this stage compounds were at $1 \mathrm{x}$ desired concentration, and 915 DMSO was at $1 \%$ concentration). For a non-reactive negative control, $20 \mu \mathrm{L}$ of $1 \mathrm{x}$ 916 kinase assay buffer containing no c-Raf was added to the mixture instead. The plate 917 was incubated at $30{ }^{\circ} \mathrm{C}$ for $45 \mathrm{~min}$. After the 45 -min reaction, $50 \mu \mathrm{L}$ of kinase 918 Kinase-Glo Max reagent (Promega, cat. no. V6071) was added to each well, and the 919 plate was incubated at room temperature for $15 \mathrm{~min}$, at dark. The plate was read with 920 a microplate reader (BMG Labtech) for luminescence reading. The luminescence 921 reading value measures the levels of ATP remaining, which is inversely related to 922 kinase activity. The non-reactive negative control read value, indicating the level of 923 initial added ATP, subtracted the level of ATP remaining (the luminescence reading) 924 for the value of consumed ATP in the reaction that represents a kinase activity. 


\section{In vitro pull-down assays}

We performed in vitro pull-down assays to test the compounds' influence on the HTT-LC3 interactions. The purified HTTexon1 (with the indicated tags), full-length proteins and the control proteins were incubated with amylose resin (New England BioLabs, cat. no. E8021L) at $4{ }^{\circ} \mathrm{C}$ for $30 \mathrm{~min}$. Immobilized amylose resins were then washed three times with HBS (20 mM HEPES pH7.5, $150 \mathrm{mM} \mathrm{NaCl}, 0.05 \%$ Tween-20). The resulting amylose resins containing about $10 \mu \mathrm{g}$ of MBP-fused proteins were incubated with the indicated compounds $(1 \mu \mathrm{M}$ for 1005 and $100 \mathrm{nM}$ for AN2) or the DMSO control at the same volume in $300 \mu \mathrm{l}$ of $\mathrm{HBS}$ at $4{ }^{\circ} \mathrm{C}$ for $1 \mathrm{~h}$ using sample mixer. $40 \mathrm{\mu g}$ of purified LC3B protein were then added and incubated at $4{ }^{\circ} \mathrm{C}$ for another $2 \mathrm{~h}$ using sample mixer. The resin-bound proteins were eluted with $40 \mu \mathrm{l}$ maltose buffer (10 mM maltose, $20 \mathrm{mM}$ HEPES, $150 \mathrm{mM} \mathrm{NaCl}, \mathrm{pH}$ 7.5) and then added with $20 \mu \mathrm{l}$ SDS-PAGE sample loading buffer. Samples were then analyzed by SDS-PAGE and Western-blots.

GST pulldown was performed as the same procedures described above, except that GST-fused LC3B was immobilized onto magnetic conjugated GST mouse mAb beads (Cell Signaling Technology, cat.no.11847S) and eluted with SDS-PAGE protein loading buffer by vortex according to the instruction manual.

\section{Imaging-based autophagy assays}

Analysis of GFP-LC3 puncta for measuring autophagosomes: HeLa cells stably expressing GFP-LC3 were generated by transfection of pEGFP C1-LC3, and positive clones were selected by $500 \mu \mathrm{g} / \mathrm{ml} \mathrm{G} 418$. The cells were then treated with vehicle (DMSO, 0.1\%), 1005, or AN2 for the indicated concentration, chloroquine (CQ, 20 $\mu \mathrm{M})$ treatment was used as a control. After 24 hours, cells were fixed in $4 \%$ paraformaldehyde (PFA) for $10 \mathrm{~min}$. Images were acquired with confocal microscopy (Leica SP8) by the observer blinded to the identity of the slides. The number and size of GFP vesicles per cell was determined by Image $J$ software. Images were processed with the despeckle function to decrease the noise, and a threshold was set to highlight puncta. Cells were selected by the freehand drawing tool. The analyze-particle function was used for the sizes and numbers of GFP puncta.

The mRFP-GFP-LC3 assay: this assay allows us to monitor autophagosome synthesis and maturation/fusion by labelling autophagosomes (green and red) and autolysosomes (red), since the low lysosomal $\mathrm{pH}$ in autolysosomes quenches the GFP signals ${ }^{32}$. HeLa cells stably expressing mRFP-GFP-LC3 ${ }^{32}$ were treated with vehicle (DMSO, $0.1 \%$ ), 1005, or AN2 for the indicated concentration, bafilomycin-A1 (bafA1, $10 \mathrm{nM}$ ) treatment was used as a control. After 24 hours, cells were fixed in $4 \%$ PFA for 10 min. Images were acquired with confocal microscopy (Leica SP8) by the 
962

963

964

965

966

967

968

969

970

971

972

973

974

975

976

977

978

979

980

981

982

983

984

985

986

987

988

989

990

991

992

993

994

995

996

997

998

observer blinded to the identity of the slides. The green and red single channel images were analyzed by Image $\mathrm{J}$ to quantify green and red puncta in the same way as in the GFP-LC3 assay described above.

\section{Detection of long-lived proteins by click-chemistry}

As an indicator of autophagy activity, the degradation of long-lived proteins was measured similarly as previously reported ${ }^{33}$. Basically, the HeLa cells with $70 \sim 80 \%$ confluency in a 6-well plate were washed with warm PBS and cultured in Met-free DMEM (ThermoFisher Scientific, cat. no.21013) added with dialyzed FBS for $1 \mathrm{~h}$ to deplete intracellular free Met reserves. The Met analog L-AHA $(50 \mu \mathrm{M})$ was then added to label the proteins for $18 \mathrm{~h}$. After labeling, the cells were washed with PBS and cultured in regular culture medium containing 10x L-Met ( $2 \mathrm{mM})$ for $2 \mathrm{~h}$ to chase out short-lived proteins. The cells were then treated with the compounds versus the DMSO controls for $6 \mathrm{~h}$ before cell lysis and protein extraction. For the starvation sample, the culture medium was replaced with EBSS (ThermoFisher Scientific, cat. no. 24010043) for $6 \mathrm{~h}$. The protein lysates were then used for the click reaction by the Click-it reaction kit (Click Chemistry tools, cat. no. C1001) following manufacturer's instructions, and the remaining L-AHA containing long-lived proteins were then conjugated with biotin. These proteins were then analyzed by electrophoresis and detected by the HRP-conjugated streptavidin (Beyotime, cat. no. A0303).

Immunofluorescence and caspase-3 imaging

For immunofluorescence of cultured cells, cells were fixed in 4\% PFA for 10 min after washing with $1 \times$ PBS for three times, and then washing and permeabilized in $0.5 \%$ (vol/vol) TritonX-100 for $10 \mathrm{~min}$. The cells were then blocked in blocking buffer ( $4 \%$ $\mathrm{BSA}+0.1 \%$ (vol/vol) Triton X-100 in 1 $\mathrm{PBS}$ ) for $30 \mathrm{~min}$ and incubated overnight at $4{ }^{\circ} \mathrm{C}$ with primary antibodies, and then washed three times with blocking buffer and incubated with secondary antibody at room temperature for $1 \mathrm{~h}$. Coverslips were then washed three times, stained with $0.5 \mathrm{mg} / \mathrm{ml} \mathrm{DAPI}$ for $5 \mathrm{~min}$ at room temperature, and then mounted in vectashield mounting medium (Vector, cat.no. $\mathrm{H}-1002$ ). Images were taken by Zeiss Axio Vert A1 confocal microscopes and analyzed blindly by ImageJ for co-localization and TUBB3 quantifications. For co-localization experiments of transfected HeLa cells (Fig. 4c), the GFP signals were used to detect GFP-LC3B, and anti-His was used to detect HTTexon1-MBP-His proteins. Empty vector transfected cells were imaged to ensure the specificity of the signals. The co-localization was analyzed by calculating the ratio between overlapping puncta and the HTT (red) puncta for each cell, and the puncta numbers were counted blindly. For co-localization experiments of STHdh ${ }^{\text {Q111/Q111 }}$ cells, the endogenous $\mathrm{mHTT}$ protein was stained with the HTT antibody (Millipore, cat. no. MAB2166), and autophagosomes were stained 
with the LC3B antibody (ThermoFisher Scientific, cat. no. 700712), which preferentially detects LC3-II ${ }^{38}$. The co-localization was analyzed by ImageJ to calculate the ratio between overlapping pixels and the HTT (red) positive pixels, because the signals of the endogenous proteins were more dispersed and could not be counted accurately. For TUBB3, the total area of TUBB3 signals and the DAPI counts were analyzed by ImageJ. The former is then divided by the latter to calculate the averaged area of TUBB3 in each neuron as an index for neurodegeneration in vitro.

For caspase- 3 activity measurements of the iPSC-derived neurons, the NucView 488 caspase- 3 dye (Biotium, cat. no. 30029) was used for the caspase 3 activity detection as an indicator for apoptosis. The images were then taken every $3 \mathrm{~h}$ using the Incucyte technology (Essen Bioscience, IncuCyte FLR), which takes images of 4 different fields in each well inside the cell culture incubator. The quantification was performed by the Incucyte $2011 \mathrm{~A}$ software, which identified the green fluorescent puncta and quantified the fluorescent object count per field. The 4 fields per well were quantified and averaged, and 4 independent wells were used for statistical analysis.

\section{Antibodies}

Antibodies used for Western-blots, HTRF and/or immunofluorescence/immunohistochemistry are as follows: the HTT antibodies $2 \mathrm{~B} 7^{39}$, $\mathrm{ab} 1^{40}$ and $\mathrm{MW} 1^{41}$ have been described previously; commercially purchased antibodies include HTT antibody 2166 (Millipore, cat. no. MAB2166), anti-polyQ antibody 3B5H10 (Sigma, cat. no. P1874), anti-HTT antibody (D7F7)XP (Cell Signaling Technologies, cat. no. 5656s), anti- $\beta$-tubulin (Abcam, cat. no. ab6046), anti-TUBB3 (Biolegends (previously Covance), cat. no. 801202), anti-ATXN3 (Millipore, cat. no. MAB5360); anti-Gapdh (Proteintech, cat. no. 60004-1), anti-NBR1 (ThermoFisher Scientific, cat. no. PA5-54660), anti- $\beta$-actin (Beyotime, cat. no. AA128); anti-TBP (Abcam, cat. no. ab818); anti-P62 (ThermoFisher Scientific, cat. no. PA5-27247); anti-spectrin (Millipore, cat. no. MAB1622); anti-Ncoa4 (Santa cruz, cat.no. sc-373739); anti-GST (ProteinTech, cat. no. HRP-66001); anti-GFP (Cell Signaling Technologies, cat. no. 2956); anti-MBP (ProteinTech, cat. no. 15089-1-AP); anti-His (Beyotime, cat. no. AH367); anti-BUBR1 (BD Transduction, cat.no, 612503); anti-phospho-p44/42 MAPK (ERK1/2) and anti-phospho-MEK1/2 in the Phospho-Erk1/2 Pathway Sampler Kit (Cell Signaling Technology, cat.no. 9911); anti-LC3B (ThermoFisher Scientific, cat.no. PA1-16930 (for Western-blot) \& cat. no. 700712 (for immunofluorescence)). All the antibodies used for immunofluorescence in this study have been validated by knock-down experiments. All the HTT, polyQ and ATXN3 antibodies used for HTRF and/or Western-blots have been validated by 
1036 knock-down experiments and by comparing the signals from different genotypes in

1037 previous studies from us and others. All the other antibodies have been validated by

1038 previous literature or the vendor.

\section{Compound detection in vivo in brain tissue from ip-injected mice}

1040 The experiments were performed by the SIM-Servier joint laboratory. The mice ip-injected with DMSO or the indicated compounds were anesthetized by chloral hydrate (200 $\mu \mathrm{L} / \mathrm{kg}$ of $10 \%$ stock) at indicated time points, and the heart blood was collected by vacuum blood collection tubes. The heart blood samples were further spun at 10,000 rpm for 5 min to generate the heart plasma. The mice were then perfused with 1X PBS to remove the blood. The mice were then sacrificed and the brain samples were dissected. 5 times of volume of methanol: acetonitrile (50: 50, $\mathrm{vol} / \mathrm{vol}$ ) were added to each sample, which was then homogenized. Following ultrasonic treatment for $15 \mathrm{~min}$, the homogenates were centrifuged for $5 \mathrm{~min}$, then 20 $\mu \mathrm{L}$ supernatant liquid was mixed with $20 \mu \mathrm{L}$ water for 30 s before injection. Linear range of 1005 was $10-30000 \mathrm{ng} / \mathrm{mL}$, and the linear range of AN2 was 0.3-10000 $\mathrm{ng} / \mathrm{mL}$. The LC-MS/MS analyses were performed on an Acquity ultra performance liquid chromatography (UPLC) system (Waters Corporation, Milford, MA, USA) coupled to a Xevo TQ-S mass spectrometer (Waters Corporation, Milford, MA, USA). Chromatographic separation was performed using an Acquity UPLC BEH C18 (1.7 $\mu \mathrm{m} 2.1 \times 50 \mathrm{~mm}$ ) column supplied by Waters at a flow of $0.5 \mathrm{~mL} / \mathrm{min}$. Gradient elution was used with a mobile phase composed of solvent $A$ (water containing $0.1 \%$ formic acid and $5 \mathrm{mM} \mathrm{NH}_{4} \mathrm{AC}$ ) and solvent $\mathrm{B}$ (acetonitrile: methanol (9:1, vol/vol) containing $0.1 \%$ formic acid).

\section{Proteomics analysis}

Samples were analyzed on Orbitrap Fusion Lumos mass spectrometers (Thermo Fisher Scientific, Rockford, IL, USA) coupled with an Easy-nLC 1000 nanoflow LC system (Thermo Fisher Scientific). Dried peptide samples were re-dissolved in Solvent $A(0.1 \%$ formic acid in water $)$ and loaded to a trap column $(100 \mu \mathrm{m} \times 2 \mathrm{~cm}$; particle size, $3 \mu \mathrm{m}$; pore size, $120 \AA$; SunChrom, USA) with a max pressure of $280 \mathrm{bar}$ using Solvent A, then separated on a $150 \mu \mathrm{m} \times 15 \mathrm{~cm}$ silica microcolumn (particle size, $1.9 \mu \mathrm{m}$; pore size, $120 \AA$; SunChrom, USA) with a gradient of $5-35 \%$ mobile phase $B$ (acetonitrile and $0.1 \%$ formic acid) at a flow rate of $600 \mathrm{~nL} / \mathrm{min}$ for $75 \mathrm{~min}$. The FAIMS device was placed before the mass spectrometer. FAIMS separation was performed with the following settings: inner electrode temperature $=100{ }^{\circ} \mathrm{C}$, outer electrode temperature $=100{ }^{\circ} \mathrm{C}$, carrier gas flow $=4.6 \mathrm{~L} / \mathrm{min}$, Dispersion Voltage $=-5000 \mathrm{~V}$, entrance plate voltage $=250 \mathrm{~V}$. The FAIMS carrier gas is $\mathrm{N}_{2}$ only. The noted CVs were applied to the FAIMS electrodes. Each of the selected CVs was applied to sequential 
1073 survey scans and MS/MS cycles (1s); the MS/MS CV was always paired with the 1074 appropriate CV from the corresponding survey scan. For detection with Fusion or 1075 Fusion Lumos mass spectrometry, a precursor scan was carried out in the Orbitrap by 1076 scanning $\mathrm{m} / \mathrm{z} 300-1400$ with a resolution of 120,000. The most intense ions selected 1077 under top-speed mode were isolated in Quadrupole with a $1.6 \mathrm{~m} / \mathrm{z}$ window and 1078 fragmented by higher energy collisional dissociation (HCD) with normalized collision 1079 energy of $30 \%$, then measured in the linear ion trap using the rapid ion trap scan rate. 1080 Automatic gain control targets were $5 \times 10^{5}$ ions with a max injection time of $50 \mathrm{~ms}$ for 1081 full scans and $1 \times 10^{4}$ with $35 \mathrm{~ms}$ for MS/MS scans. Dynamic exclusion time was set at $108218 \mathrm{~s}$. Data were acquired using the Xcalibur software (Thermo Scientific).

1083 Rawfiles were searched against the human National Center for Biotechnology 1084 Information (NCBI) Refseq protein database (updated on 04-07-2013, 32,015 entries) 1085 by Mascot 2.3 (Matrix Science) implemented on Proteome Discoverer 2.2 (Thermo 1086 Scientific). The mass tolerances were $20 \mathrm{ppm}$ for precursor and $0.5 \mathrm{Da}$ for product 1087 ions for Fusion Lumos. Up to two missed cleavages were allowed. The search engine 1088 set cysteine carbamidomethylation as a fixed modification and $\mathrm{N}$-acetylation, 1089 oxidation of methionine as variable modifications. Precursor ion score charges were 1090 limited to $+2,+3$, and +4 . The data were also searched against a decoy database so 1091 that protein identifications were accepted at a false discovery rate of $1 \%$. Label-free 1092 protein quantifications were calculated using a label-free, intensity-based absolute 1093 quantification (iBAQ) approach.

1094 Proteins with at least 2 unique peptides with 1\% FDR at the peptide level and 1095 Mascot ion score greater than 20 were selected for further analysis. The file used for 1096 protein inference and protein FDR calculation was derived from Mascot search results, 1097 and the peptide spectrum match (PSM) was filtered via Percolator and customized 1098 parameters, and then the proteins were assembled. The protein FDR was calculated 1099 depending on the ratio of NPD (the number of assembled proteins from decoy 1100 database searches) and NPT (the number of assembled proteins from target 1101 database searches). The FOT was used to represent the normalized abundance of a 1102 particular protein across samples. FOT was defined as a protein's iBAQ divided by the 1103 total $\mathrm{iBAQ}$ of all identified proteins within one sample. The FOT was multiplied by $10^{5}$ 1104 for the ease of presentation. Only the proteins detection in all compared samples 1105 were utilized for comparison.

\section{Behavioral and lifespan experiments in HD Drosophila models}

1107 For behavioral experiments, we placed 15 age-matched virgin female flies in an 1108 empty vial and tapped them down. The percentage of flies that climbed past a 1109 7-cm-high line after $15 \mathrm{~s}$ was recorded. The mean of five observations is plotted for 
each vial on each day, and data from multiple vials containing different batches of flies were plotted and analyzed by two-way ANOVA tests. The flies were randomly placed into each tube. For lifespan measurements, we placed 75 age-matched virgin female flies in an empty plastic vial and recorded the survival situation for each vial on each day. For both behavioral and lifespan measurement experiments, the person who performed the experiments were blinded to the drugs fed until data analysis.

\section{Behavioral experiments in HD mouse models}

All the behavioral experiments were performed during the light phase and the experimenters were blinded to the compound treatment and the genotype of each mouse. Both males and females have been used. All the mice were kept in the behavioral test room in dim red light for $1 \mathrm{~h}$ before starting the experiments. For rotarod experiments, mice were pre-trained on 3 consecutive days on the rotarod rotating at $4 \mathrm{rpm}$ for $2 \mathrm{~min}$. Mice were then tested for five days at an accelerating speed ranging from 4 to $40 \mathrm{rpm}$ within $2 \mathrm{~min}$. Each performance was recorded as the time in seconds spent on the rotating rod until falling off or until the end of the task. Each test included three repetitions with an inter-trial interval of $60 \mathrm{~min}$ in order to reduce stress and fatigue, and the means from these three runs were analyzed for each mouse. The balance beam test was run using a $2 \mathrm{~cm}$ thick meter stick suspended from a platform on both sides by metal grips. The total length is $100 \mathrm{~cm}$. There was a bright light at the starting point and a dark box with food at the endpoint. The total time for each mouse to walk through the bean was recorded. For gripping force measurements, mice were allowed to grip the metal grids of a grip meter (Ametek Chatillon) with their forelimbs, and they were gently pulled backwards by the tail until they could no longer hold the grids. The peak grip strength observed in 10 trials was recorded.

\section{Statistics}

To ensure to reach a statistical power $>0.8$, power analyses were performed for each assay based on estimated values by PASS 16 (https://www.ncss.com/software/pass/) before experiments. Estimation was based on our previously published results on similar experiments and preliminary experiments. The effect size was also estimated by Cohen's $d$, two means divided by the standard deviation for the data. The power analysis suggested $n \geq 3$ for mHTT level measurements and $n \geq 5$ for behavioral experiments. In all the experiments we performed, we have used a larger $n$ than these numbers in case the effect was smaller than preliminary results, and we also performed post-experiment power analyses to ensure that power $\geq 0.8$ for all the significant differences. Statistical comparisons between two groups were conducted by the unpaired two-tailed $t$ tests. 
1147 Statistical comparisons among multiple groups were conducted by one-way ANOVA

1148 tests and post-hoc tests for the indicated comparisons (Dunnett's tests for comparison

1149 with a single control, and Bonferroni's tests for comparisons among different groups).

1150 Statistical comparisons for series of data collected at different time points were

1151 conducted by two-way ANOVA tests. The similarity of variances between groups to be

1152 compared was tested when performing statistics in GraphPad Prism 7 and Microsoft

1153 Excel 2016. Normality of data sets was assumed for ANOVA and tests, and was

1154 tested by Shapiro-Wilk tests. When the data were significantly different from normal

1155 distribution, nonparametric tests were used for statistical analysis. All statistical tests

1156 were unpaired and two-tailed.

1157

\section{Reference for Methods}

1159

38 Hancock, M. K., Hermanson, S. B. \& Dolman, N. J. A quantitative TR-FRET

39 Weiss, A. et al. Single-step detection of mutant huntingtin in animal and human plate reader immunoassay for measuring autophagy. Autophagy 8, 1227-1244,

41 Ko, J., Ou, S. \& Patterson, P. H. New anti-huntingtin monoclonal antibodies:

\section{Acknowledgements}

1174 The authors would like to thank Drs. Junmei Lu, Min Jiang, Linyun Liu and Qian Huang for their technical support of mouse behavioral experiments. Dr. Yanhui $\mathrm{Xu}$ for technical supports of the protein purification. Dr. Hexige Saiyin for the help with obtaining human patient fibroblasts. The authors would also like to thank the following funding supports: National Key Research and Development Program of China (2016YFC0905100), National Natural Science Foundation of China (8192500069, 81870990, 31961130379, 91649105, 31470764, 91527305, and 61505032), Science and Technology Commission of Shanghai Municipality (18410722100), Natural Science Foundation of Shanghai (19ZR1405200), Shanghai Municipal Science and Technology Major Project (No.2018SHZDZX01) and ZJLab, and Hereditary Disease Foundation. 


\section{Author Contribution}

$\mathrm{B}$ Lu perceived the idea, initiated the project, designed experiments, analysed data and wrote the manuscript. $Y$ Fei and $C$ Zhu performed the OI-RD screening and $K_{\text {on }} / K_{\text {off }}$ measurements with data analysis. Y Ding, Z Wang, J Li, C Gao performed protein purification, in vitro pull-down and structural biology experiments with data analysis. With the help from others for blinding, Z Li performed the HTT measurements in cells and in mouse models, the mouse phenotype experiments, the autophagy-related mechanistic experiments, the control protein measurements, and neurotoxicity measurments. $C$ Wang replicated Z Li's HTT measurement and autophagy-related mechanistic experiments, and performed additional HTT measurement and phenotypic experiments in HD fly models, patient iPSC-derived neurons and MEFs, as well as all the measurements of other polyQ proteins and all the MST experiments. $Y$ Dang provided the compound library for the screen. T Sha and $C$ Ding performed proteomics experiments and analysis. S Luo and $Y$ Yang performed the measurements of autophagy function. L Ma, Y Sun and $\mathrm{J}$ Wang provided and characterized the patient cell lines. $X$ Sun did the initial subcloning of full-length HTT and found the explanation for the observed "hook effects". C Lu (biostatistician) performed biostatistical analysis. M Difiglia and $Y$ Mei helped designing the experiments and interpreting the data.

1203

\section{Data Availability Statement}

1213 The protein structure data has been uploaded to the PDB database with entry number

1214 6J04. Source data for all figure plots are provided with the paper. The full gel blots and the 1215 proteomics datasets have been provided in supplementary information. The data that support the findings of this study are available from the corresponding authors upon reasonable request.

\section{Extended data figure/table legends}

\section{Extended Data Fig. 1. Protein purifications.}

a) SDS-PAGE and linear mode MALDI-TOF mass spectrometry analysis of the expression and purification of recombinant LC3B protein. For SDS-PAGE, lane 1, the 
whole cell lysate before induction; lane 2, the whole cell lysate after induction; lane 3 , the supernatants of induced cells; lane 4, the flow through fraction of Ni-NTA chromatography; lane 5, the eluates of Ni-NTA chromatography (GST-His8-LC3); lane 6, the eluates of GST-His8 tag removed LC3B by TEV protease; lane 7, the eluates of size exclusive chromatography; lane 8 , molecular weight marker. The obtained mass spectrometry $\mathrm{m} / \mathrm{z}$ peak of recombinant LC3B is 14660.811 , consistent with theoretical calculations

b) Structural alignment of purified recombinant LC3B $\triangle$ G120 (PDB: 6J04, yellow) with published LC3B structure (PDB: 1UGM, cyan) by Pymol.

c-d) SDS-PAGE and linear mode MALDI-TOF mass spectrometry analysis of the HTTexon1 proteins. For HTTexon1-Q72-MBP SDS-PAGE, lane 1, the supernatants of induced cells; lane 2, the insoluble fraction of induced cells; lane 3, the flow through fraction of Ni-NTA chromatography; lane 4, the eluates of Ni-NTA chromatography; lane 5 , the eluates of size exclusive chromatography; lane 6 , molecular weight marker. For HTTexon1-Q25-MBP SDS-PAGE, lane 1, molecular weight marker; lane 2, the induced cell lysate; lane 3 , the supernatant fraction of induced cells; lane 4 , the flow through fraction of Ni-NTA chromatography; lane 5 and 6 , the eluates of Ni-NTA chromatography; lane 7 and 8 , the eluates of size exclusive chromatography. The obtained mass spectrometry $\mathrm{m} / \mathrm{z}$ peak of HTTexon1-Q72-MBP and HTTexon1-Q25-MBP are 64225.946 and 58228.893 , consistent with theoretical calculations.

e) Left and middle panels: size exclusive chromatography of the recombinant full-length HTT-Q73 (flHTT-Q73) and HTT-Q23 (flHTT-Q23) proteins using Superose 6 5/150 GL. The major peak fractions were then collected pooled together for the SDS-PAGE analysis (right panel).

f) SDS-PAGE analysis of purified MBP-His8 (MBP), sfGFP (GFP), and Rpn10 proteins.

Extended Data Fig. 2. Negative controls for OI-RD measurements and validation of the compounds' interaction with HTT and LC3 by MST.

a) Similar to Fig. 1c-e, but for negative control proteins MBP-His8 (MBP), superfolder GFP (sfGFP), and ubiquitinated-Rpn10 (Ub-Rpn10). Association-dissociation curves of surface immobilized compounds 8F20 and 1005 with these proteins were measured by OI-RD, and no compound-protein interactions were detected. For all association-dissociation curves, vertical dash lines mark the starts of association and dissociation phases of the binding event.

b) Binding of 1005 and 8F20 to full-length HTT-Q73 (flHTT-Q73, black dots) or LC3B (red dots) in standard treated capillaries measured by MST. The compound bound protein fractions (bound / total) were calculated by the MST signals $\left(F_{\text {norm }}\right)$ at each compound

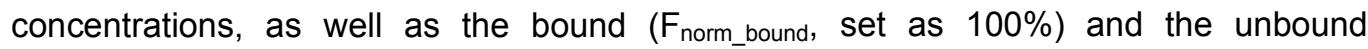
( $F_{\text {norm_unbound }}$, set as $\left.0 \%\right) M S T$ signals: bound $/$ total $=\left(F_{\text {norm }}-F_{\text {norm_unbound }}\right) /\left(F_{\text {norm_bound }}-\right.$ $\left.F_{\text {norm_unbound }}\right) \times 100 \%$. The fitted curves and calculated $K_{d}$ values by the Nanotemper analysis software (1.5.41) for flHTT-Q73 and LC3B were indicated in each panel. Consistent with the OI-RD measurements (Fig. 1e), no binding was observed for the flHTT-Q23 protein (blue dots). The MST experiments were repeated $>3$ times and showed consistent results.

c) Similar to b), except testing the compounds indicated in the X-axis. MST measurements 
of the binding of indicated compounds to full-length HTT-Q73 (flHTT-Q73), full-length HTT-Q23 (flHTT-Q23) and LC3B in standard treated capillaries. The proteins tested were indicated in the legends.

d) Similar to Fig. 1c-e, but plotting the association-dissociation curves of surface immobilized compound AN2 with full-length HTT-Q73 (Q73), or full-length HTT-Q23 (Q23), or LC3B, or the negative control proteins MBP-His8 (MBP), superfolder GFP (sfGFP), and Rpn10.

For all association-dissociation curves, vertical dash lines mark the starts of association and dissociation phases of the binding event. The red dash lines are global fits to a Langmuir reaction model with the global fitting parameters listed at the bottom of each plot. No binding signals were observed for full-length HTT-Q23 proteins, and thus the parameters were not presented.

e) Cell viability measurement of cultured HD neurons measured by the CellTiter-glo assay. No toxicity was observed within the concentration range presented in Fig. 2, although the compound $8 \mathrm{~F} 20$ became toxic to the cells when the concentration reached $300 \mathrm{nM}$.

\section{Extended Data Fig. 3. mHTT lowering effects by mHTT-linker compounds could be} detected by multiple antibodies and were dependent on autophagy.

a) Representative Western-blots (HTT detected by the 2166 antibody) and quantifications of compound-treated cultured cortical neurons from $\mathrm{Hdh}^{\mathrm{Q} 7 / \mathrm{Q} 140} \mathrm{HD}$ knockin mice. The neurons were treated with the indicated compounds (100 nM for 1005, 8 F20 and AN1; 50 $\mathrm{nM}$ for AN2) with or without the autophagy inhibitor $\mathrm{NH}_{4} \mathrm{Cl}$ (upper panels) or chloroquine (lower left), or the autophagy activator rapamycin (lower right). Same amount of culture medium was added in the controls (upper panels). The statistical analysis was performed by one-way ANOVA with post-hoc Dunnett's tests, and the F/degree of freedom/post-hoc $p$ values have been indicated in each bar plot.

b) Western-blots using indicated HTT/polyQ antibodies for samples from cultured cortical neurons treated with the indicated compounds: 1005 (100 nM), 8F20 (100 nM), AN2 (50 $\mathrm{nM}$ ). The HTT gel blots presented in Fig. 2d (right panel) were cropped from first four blots. The low molecular weight bands were run out in these blots so that the wtHTT and MHTT could be better separated. Note that the weak bands just above $250 \mathrm{kDa}$ in the first two blots were leftover signals from the Spectrin blotting. The Spectrin signals were too strong to be stripped completely.

c) Western-blots using the antibody MW1 or 3B5H10 that detects mHTT specifically. The relatively low molecular weight proteins were kept. No increase of potential polyQ-containing $\mathrm{mHTT} \mathrm{N}$-terminal fragments was observed.

d) HD patient iPSC-derived striatal neurons (Q47) were treated with the indicated compounds (100 $\mathrm{nM}$, with $0.1 \%$ DMSO) in presence of an additional $0.1 \%$ DMSO or 10 $\mathrm{mM} \mathrm{NH}_{4} \mathrm{Cl}$, and the $\mathrm{mHTT}$ levels were measured by HTRF using the 2B7/MW1 antibody pair. All signals were normalized to the averaged signals from the DMSO control group. The statistical analysis was performed by one-way ANOVA with post-hoc Dunnett's tests, and the F/degree of freedom/post-hoc $p$ values have been indicated in each bar plot. " "***": $p<0.0001$. The post-hoc analysis was not performed if the ANOVA tests did not show significance $(p>0.05)$. 
1311

1312

1313

1314

1315

1316

1317

1318

1319

1320

1321

1322

1323

1324

1325

1326

1327

1328

1329

1330

1331

1332

1333

1334

1335

1336

1337

1338

1339

1340

1341

1342

1343

1344

1345

1346

1347

1348

1349

1350

1351

1352

1353

1354

e) The HD patient immortalized fibroblasts (Q47) were transfected with the non-targeting control siRNA (Neg siRNA) or the ATG5 siRNA (target sequence:

GCCUGUAUGUACUGCUUUA; ATG5 mRNA was knocked down to $17.7 \pm 3.0 \%, n=3$, as tested by $\mathrm{qPCR}$ ), and then after 24 hours treated with the indicated compounds (100 nM) for another 48 hours. mHTT levels were then measured by HTRF using the 2B7/MW1 antibody pair. All signals were normalized to the averaged signals from the DMSO control group. The statistical analysis was performed by one-way ANOVA with post-hoc Dunnett's tests, and the F/degree of freedom/post-hoc $p$ values have been indicated in each bar plot. “****”: $p<0.0001$. The post-hoc analysis was not performed if the ANOVA tests did not show significance. The Western-blot of LC3 confirmed the partial inhibition of autophagy in the ATG5 knockdown cells.

f) Similar to e), but in wild-type (WT) or Atg5 knockout (Atg5 KO) mouse embryonic fibroblast lines (MEF) transfected with full-length mHTT (flHTT-Q73). The Western-blot of LC3 confirmed the inhibition of autophagy in the Atg $5 \mathrm{KO}$ cells.

For all panels, $\mathrm{n}$ indicates the number of independently plated wells, and bars represent mean and s.e.m.. Full-blots of cropped gels are shown in Supplementary Fig. 1.

\section{Extended Data Fig. 4. Potential influence of c-Raf and KSP pathways by treatment} of the mHTT-LC3 linker compounds.

a) Representative results (from 3 biological repeats) of the in vitro c-Raf kinase assay (see "Methods") showing that only 1005 inhibits c-Raf activity within the concentration range tested.

b) Representative Western-blots and quantifications of phospho-MEK/ERK as an indicator of Raf activities (left) and phospho-Bubr1 as an indicator of KSP inhibition (right) in cultured cortical neurons treated with indicated compounds (100 nM for 1005, 8F20, AN1, and $50 \mathrm{nM}$ for AN2) or the DMSO control.

c) Similar to b), but in immortalized HD patient fibroblasts (Q47). Note that the phospho-Bubr1 is essentially absent and too weak to quantify, indicating that KSP was not inhibited by any of the compounds at the concentration tested.

For all panels, error bars represent mean and s.e.m.. For b, all data were corrected by the loading control ( $\beta$-Tubulin) and then normalized to the averaged signal of the DMSO control group. The statistical analysis was performed by one-way ANOVA and the $\mathrm{F} /$ degree of freedom/p values have been indicated in each bar plot. The $\mathrm{n}$ number indicates the number of independently plated and treated wells.

\section{Extended Data Fig. 5. mHTT-LC3 linker compounds lowered mHTT in transgenic HD} flies.

a) Overlay between LC3B and predicted Atg8 structure showing high structural similarities. b) Transgenic flies expressing full-length HTT-Q128 driven by elav-GAL4 were fed with indicated compounds at $10 \mu \mathrm{M}$ for 6 days, and their heads were then extracted for protein lysates. mHTT were then measured by HTRF using the 2B7/MW1 antibody pair.

Each dot represents the HTRF signal from each individual sample extracted from 5 fly heads. All the data were normalized to the average of the DMSO-fed control samples. The statistical analysis was performed by one-way ANOVA and Dunnett's post hoc tests. F (4, 
31) = 15.67; “****”: $p<0.0001$.

c) 1005 (upper panel) and AN2 (lower panel) concentrations in heart plasma and brain tissues were measured by mass-spectrum at indicated time points for compound-injected mice $(0.5 \mathrm{mg} / \mathrm{kg})$. In the brain tissue, the 1005 concentrations were $\sim 20$ to $\sim 200 \mathrm{nM}$, and the AN2 concentrations were $\sim 20$ to $\sim 40 \mathrm{nM}$, close to the effective doses that were capable of lowering mHTT in cultured neurons. Data were plotted by mean and s.e.m..

\section{Extended Data Fig. 6. mHTT-LC3 linker compounds lowered mHTT in vivo in the} mouse brains.

a) Western-blots (4 mice ( 3 months old) for each group) and quantifications of $\mathrm{mHTT}$ and wtHTT in the cortices from $\mathrm{Hdh}^{\mathrm{Q7/Q140}}$ knockin mice icv-injected with the indicated compounds ( $2 \mu \mathrm{L}$ at $25 \mu \mathrm{M}$ for each mouse) for 10 days at one-dose per day. HTT were detected by Western-blots using the 2166 antibody, and the statistical analysis was performed by one-way ANOVA and post-hoc Dunnett's tests. The F/degree of freedom/post-hoc $p$ values have been indicated under each bar plot.

b) Similar to a), except that the compounds were delivered by ip-injection $(0.5 \mathrm{mg} / \mathrm{kg})$ to $\mathrm{Hdh}^{\mathrm{Q7/Q} 140}$ mice at 5 months-old for 14 days at one-dose per day.

c) Similar to b), but from striata of ip-injected mice. The mice were injected at 10 months-old for 14 days at one-dose per day.

d) Left panel: representative dot-blot results (from two technical replicates) of the protein lysates in b) using the 4C9 antibody, which preferentially detects $\mathrm{mHTT}$ aggregates ${ }^{23}$. Middle panel: quantification of the dot-blots based on the averaged signals from two technical replicates. Right panel: measurement of $\mathrm{mHTT}$ aggregates by the 4C9/4C9 HTRF assay ${ }^{23}$.

For all panels, $\mathrm{n}$ indicates the number of mice tested, and bars represent mean and s.e.m.. For quantification, two to three technical replicates were averaged for each mouse. The statistical analysis was performed by one-way ANOVA with post-hoc Dunnett's tests, and the F/degree of freedom/post-hoc $p$ values have been indicated in each bar plot.

\section{Extended Data Fig. 7. mHTT-LC3 linker compounds did not influence autophagy.}

a) HeLa cells stably expressing GFP-LC3B were treated with $2 \mu \mathrm{l}$ vehicle $(0.1 \%$ DMSO), 1005 , or AN2 for the indicated concentration for 24 hours; chloroquine (CQ, $20 \mu \mathrm{M})$ treatment was used as a control. After 24 hours, cells were fixed, and images were acquired with confocal microscopy. The number and size of GFP vesicles per cell was determined by Image $\mathrm{J}$ software ( $\mathrm{n}$ number indicated on top of each plot). For each treatment, over 20,000 puncta were quantified ( $~ 100$ puncta per cell in 226 cells). Scale bar: $10 \mu \mathrm{m}$.

b) Representative images and quantifications of the numbers of autophagosomes (green ${ }^{+}$ puncta) and autolysosomes ( (ed $^{+} ;$green $^{-}$puncta) in HeLa cells stably expressing mRFP-GFP-LC3B. Scale bar: $10 \mu \mathrm{m}$. Autophagosome numbers or sizes were not influenced by 1005 and AN2 at indicated concentrations after 24 hours treatment (or 4 hours treatment, not shown). The autophagsome fusion was also unaffected as indicated by the autolysosome number. Note that the autophagosome/autolysosome numbers/sizes were based on image analysis of the puncta, some of which may reflect multiple vesicles. 
Green vesicles are considered to be autophagosomes (GFP+ puncta) and red vesicles are considered to be both autophagosomes and autolysosomes. The number of autolysosomes (RFP+ GFP- puncta) was achieved by subtracting the number of green vesicles from that of the red vesicles. $>10,000$ puncta from 194 cells were analyzed.

c) Representative Western-blots and quantifications of HeLa cells stably expressing GFP-LC3B. The "free GFP" was generated by lysosomal cleavage, and thus the "free GFP"|"GFP-LC3B" ratio was used as an index for autophagy flux, which was unaffected by 1005 or AN2, but decreased by the autophagy flux inhibitor chloroquine (CQ).

d) Representative Western-blots and quantifications of the chase signal of long-lived proteins in HeLa cells as an indicator of autophagy flux (see "Methods"). Consistent with previous reports ${ }^{33}$, starvation reduced the long-live protein chase signal, whereas rapamycin treatment had a milder effect. The mHTT-LC3 linker compounds 1005 and AN2 showed no influence in this assay.

e) Representative Western-blots and quantifications of LC3 in cultured cortical neurons treated with the indicated compounds. Normalized LC3-II/LC3-I was used as the indicator of autophagy. In the right panels, the 1005 and AN2 concentrations were $100 \mathrm{nM}$ and 50 $\mathrm{nM}$, respectively.

f) The SQSTM1/p62 levels were tested by Western-blots for the cortical tissues from mice injected by the indicated compounds or the DMSO control. Bars indicate mean and s.e.m.. For all panels, the $n$ numbers indicated in each bar indicate the cell numbers $(a-b)$, the number of independently plated wells (c-e) or mouse numbers (f). Error bars indicate mean and s.e.m.. The statistical analysis was performed by one-way ANOVA with post-hoc Dunnett's tests for a-e, and by two-tailed unpaired $t$ tests for $f$. Note that the post-hoc tests were not performed if the ANOVA tests failed to show significance. "“***": p $<0.0001$ (post-hoc).

Extended Data Fig. 8. Investigation on the specificity of $\mathrm{mHTT}$ lowering effects of mHTT-LC3 linker compounds.

a) Representative Western-blots and quantifications of cultured cortical neurons treated with the indicated compounds. None of the proteins tested had an obvious change (> $10 \%)$.

b) Volcano plots of the proteomics analysis of cortices from ip-injected HD mice (10 m, 4 mice per group, injected for 14 days). The concentration injected was $0.5 \mathrm{mg} / \mathrm{kg}$ with 110 $\mu \mathrm{g} / \mathrm{kg}$ DMSO, and equal amount of vehicle containing DMSO was injected as the controls. Only those proteins detected in both groups of samples used for comparisons were calculated and plotted. The dots plotted for HTT were pointed by red arrows. See Supplementary Table 2 for complete datasets. The bar plots indicate the total HTT levels normalized to the DMSO control. The actual $\mathrm{mHTT}$ reduction is anticipated to be more, because the compounds reduced $\mathrm{mHTT}$ allele-selectively.

c) Similar to b), but in cultured cortical neurons (from p0 pups, 3 wells per group). See Supplementary Table 3 for complete datasets.

In all panels, the error bars represent mean and s.e.m..

Extended Data Fig. 9. mHTT-LC3 linker compounds lowered the mutant ATXN3 
1444 a) Representative Western-blots and quantifications of ATXN3 levels in a SCA3 patient 1445 fibroblast line treated with the indicated compounds. The lowering of mutant (Q74) but not wild-type (Q27) ATXN3 was observed by treatment of linker compounds tested.

b) Quantification of the GFP intensity as an indicator of polyQ-sfGFP (25Q-GFP, 38Q-GFP, 46Q-GFP, and 72Q-GFP) protein levels in transfected HEK293T cells treated with the indicated compounds using Incucyte. The lowering of 72Q-GFP, 46Q-GFP and 38Q-GFP, but not 25Q-GFP was observed. For both a) and b), the compound concentrations were $100 \mathrm{nM}$ for 1005 and AN1, and 50 $\mathrm{nM}$ for AN2. Bar plots present mean and s.e.m., and n numbers indicate independently plated wells.

c) SDS-PAGE analysis of polyQ-sfGFP proteins (25Q, 38Q, 46Q, 53Q and 72Q) purified from HEK293T cells. The protein purification methods were similar to the ones for HTT proteins.

d) Binding of 1005, AN1 and AN2 to sfGFP (GFP) or different polyQ-sfGFP (25Q-GFP, 38Q-GFP, 72Q-GFP) proteins in standard treated capillaries measured by MST, performed and analyzed similarly as in Extended Data Fig. 2b. All these compounds interact with 38Q-GFP and 72Q-GFP, but not 25Q-GFP or GFP.

e) Association-dissociation curves of surface immobilized compounds 1005, AN1 and AN2 with polyQ-sfGFP (72Q, 53Q, 46Q, 38Q and 25Q) proteins. For all association-dissociation curves, vertical dash lines mark the starts of association and dissociation phases of the binding event. The red dash curves are fits to a Langmuir reaction model with the fitting parameters listed at the bottom of each plot. No binding signals were observed for 25Q-sfGFP (25Q).

$1467 \mathrm{f}$-h) Results of mouse behavioral test performed similarly as in Fig. 5d-f, except that the mice were injected with saline $(0.9 \% \mathrm{NaCl})$ with DMSO $(110 \mu \mathrm{g} / \mathrm{kg})$ or without DMSO. The statistical analysis was performed by two-way ANOVA with post-hoc Bonferroni's tests, and the $\mathrm{F} / \mathrm{p}$ values and degrees of freedom were indicated in the table underneath each plot.

1472 In all panels, the error bars represent mean and s.e.m..

Extended Data Table 1. Summary of data on $\mathrm{mHTT}$ lowering or rescue of HD-relevant phenotypes

A summary table showing the percentage lowering of the $\mathrm{mHTT}$ or HTT levels, as well as the percentage rescue of HD-relevant phenotypes (normalized to the difference between HD and WT) in different HD models assayed by different approaches under optimal conditions. The corresponding data figures have been indicated in the middle column. The 
Figure 1

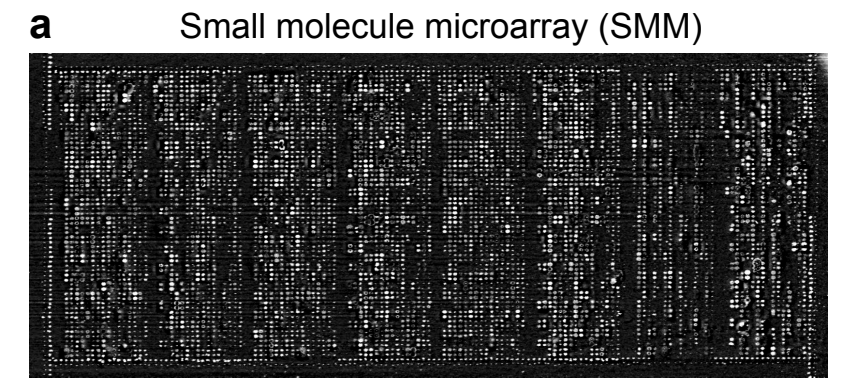

795 Known inhibitors

1527 FDA-approved drugs

1053 Natural products
Ol-RD screening signals

HTTexon1-Q72-MBP HTTexon1-Q25-MBP LC3B

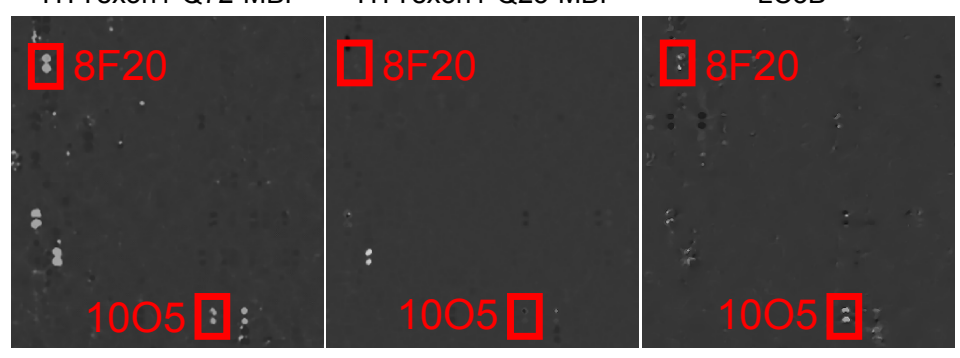

e $\quad \mathrm{K}_{\text {on }} / \mathrm{K}_{\text {off }}$ measurements for compound-HTT(full-length) interactions

C $\quad \mathrm{K}_{\mathrm{on}} / \mathrm{K}_{\text {off }}$ measurements for compound-HTTexon1 interactions
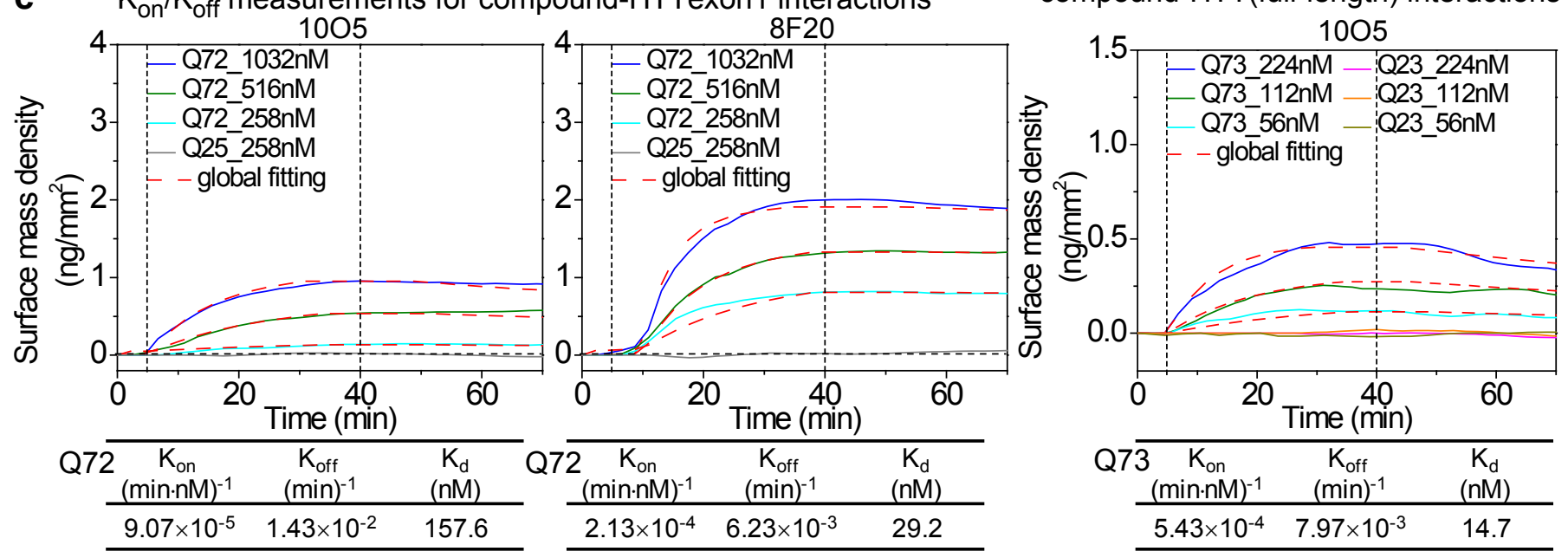

\begin{tabular}{lll}
\hline $2.13 \times 10^{-4}$ & $6.23 \times 10^{-3}$ & 29.2 \\
\hline
\end{tabular}

(
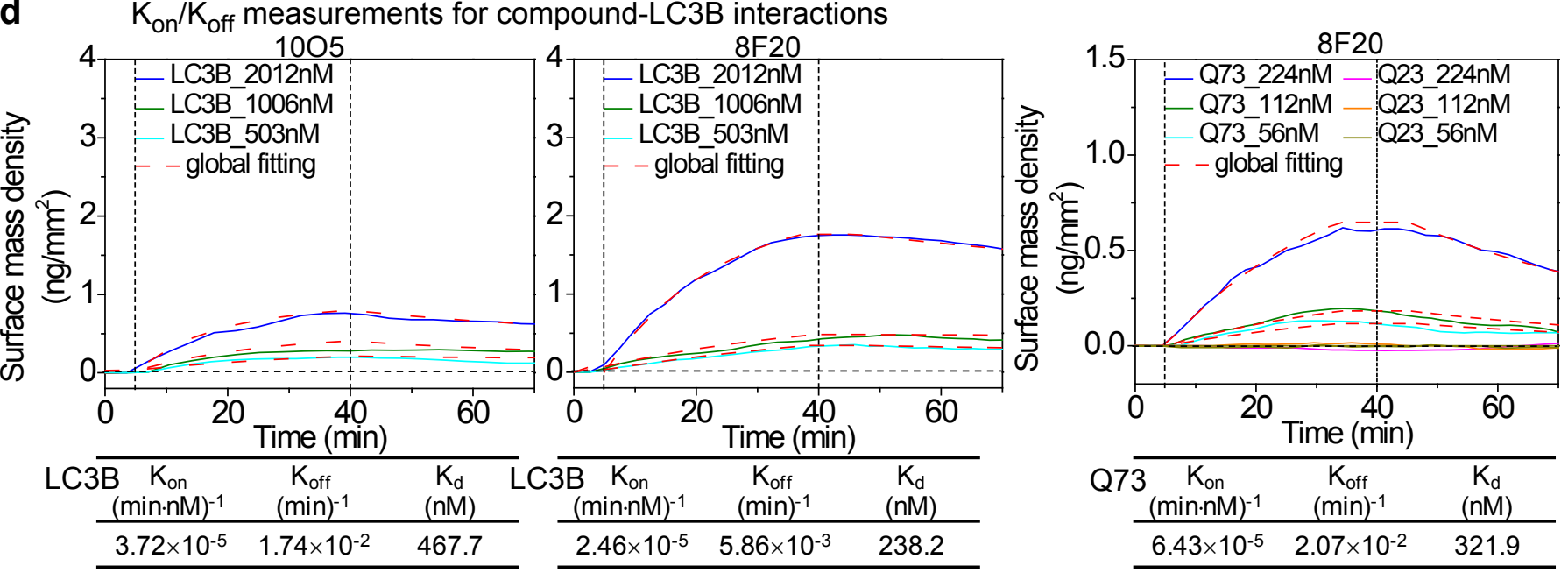


\section{Figure 2}

Cultured primary cortical neurons ( $\left.\mathrm{Hdh}^{\mathrm{Q7/Q} \mathrm{Q}^{140}}\right)$ DMSO $\frac{1005(\mathrm{nM})}{30 \quad 100 \quad 300}$ DMSO $\frac{8 \mathrm{~F} 20(\mathrm{nM})}{30 \quad 60 \quad 100}$

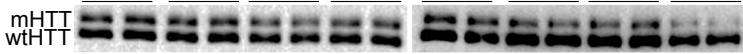
Spectrin $-\infty-\infty-\infty-\infty-\infty-\infty$

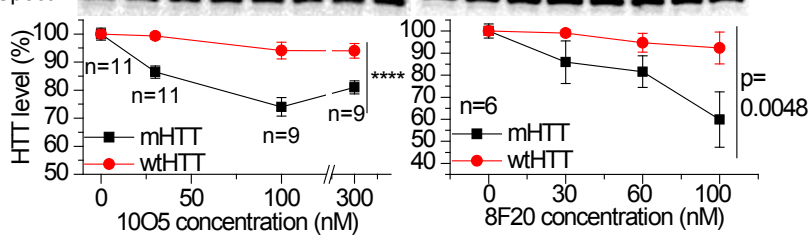

b Cultured primary cortical neurons $\left(\mathrm{Hdh}^{\mathrm{Q7/Q7}}\right)$

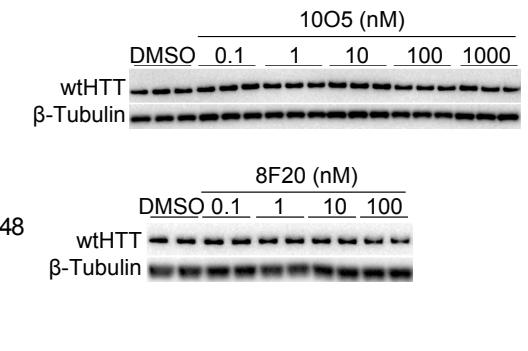

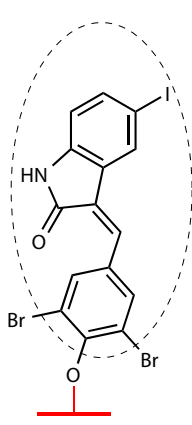

1005

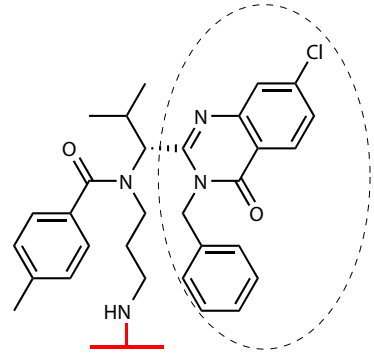

$8 \mathrm{~F} 20$

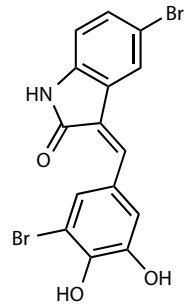

AN1<smiles>O=c1cc(-c2ccccc2)c2c(O)cc(O)cc2o1</smiles>

AN2

d Cultured primary cortical neurons $\left(\mathrm{Hdh}^{\mathrm{Q7/Q140}}\right)$

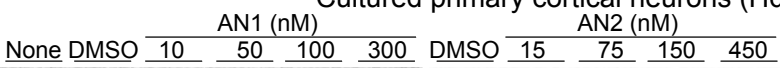

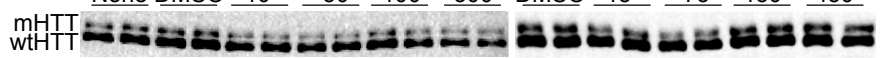

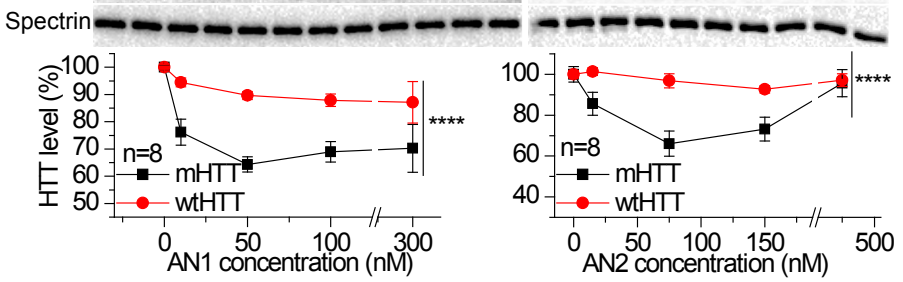

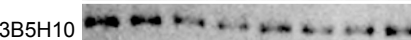
Spectrin 

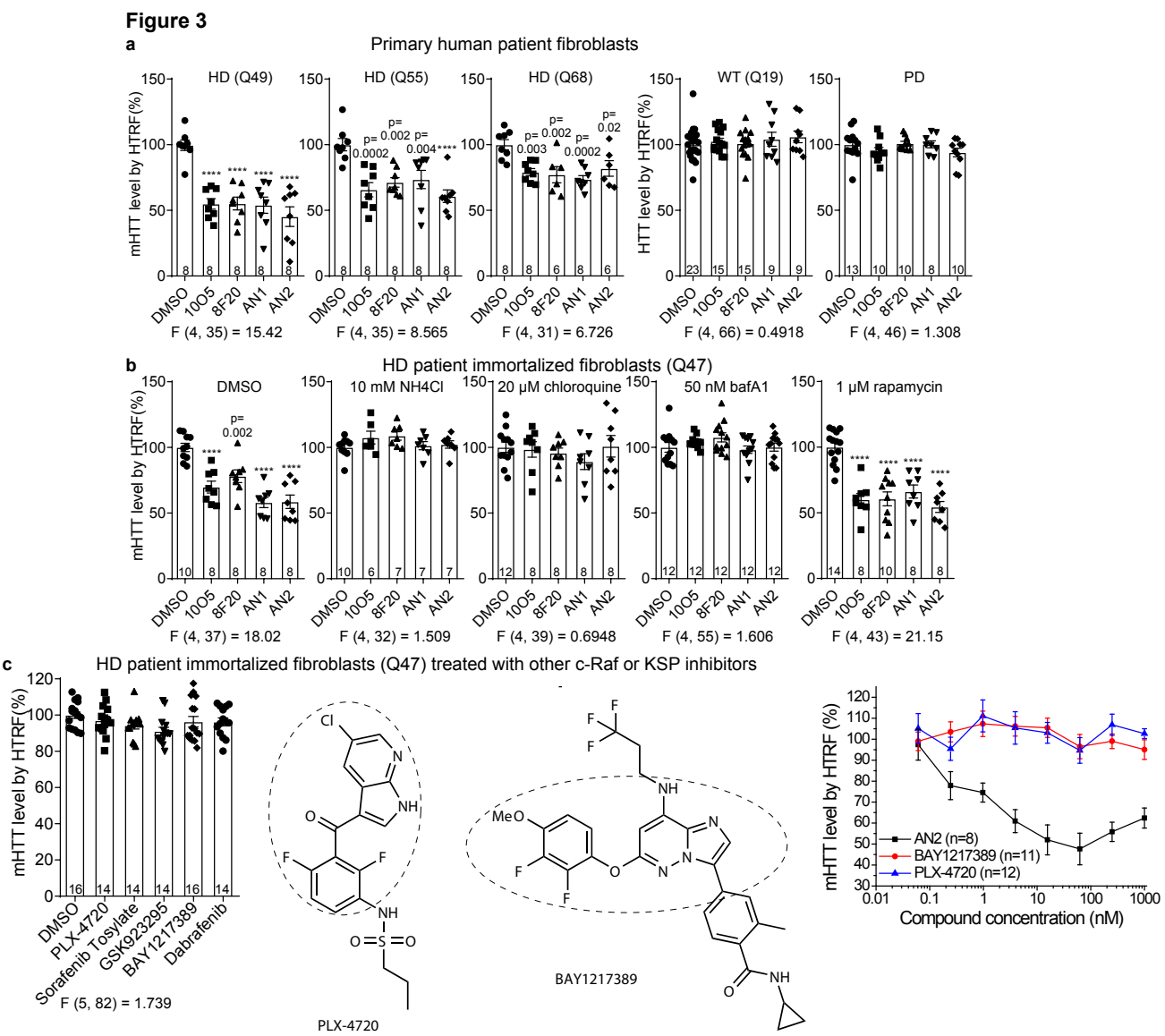
Figure 4

a

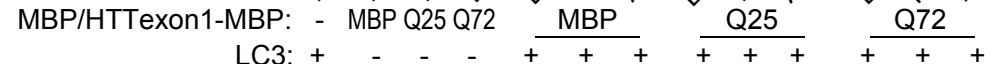
anti-LC3

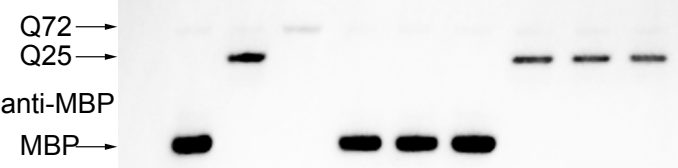

b

Compounds:

HTT: - - Q23 Q73 Q23 Q23 Q73 Q73 Q23 Q23 Q73 Q73

Full-length HTT + GST-LC3B/GST Input GST pull-down

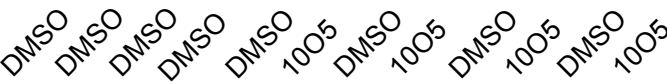
GST/GST-LC3B: GST LC3 - - GST GST GST GST LC3 LC3 LC3 LC3 anti-HTT (2166)

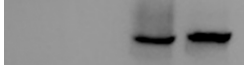

GST-LC3B $\longrightarrow$ anti-GST GST-

$-$

Input

$---$

GST pull-down

Compounds:

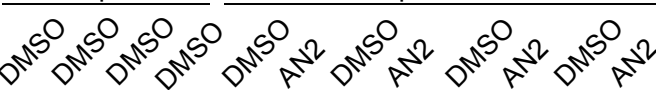

HTT:

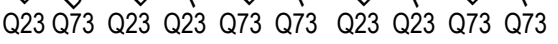

GST/GST-LC3B: GST LC3

anti-HTT (2166) GST GST GST GST LC3 LC3 LC3 LC3

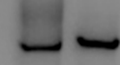

C Transfected HeLa cells (HTTexon1/LC3) GFP-LC3B+DMSO Q72-His+DMSO Q25-His+DMSO
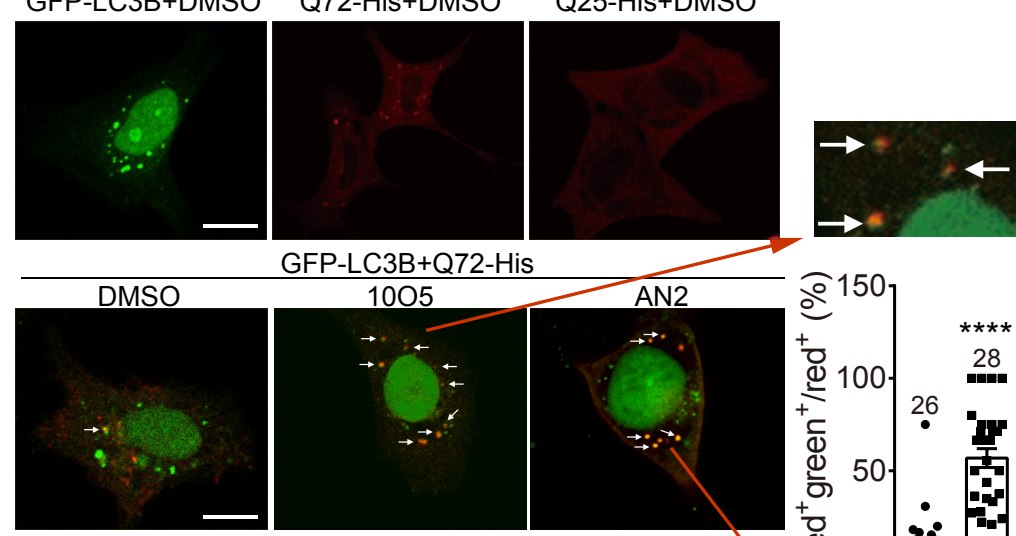

GFP-LC3B+Q72-His 1005
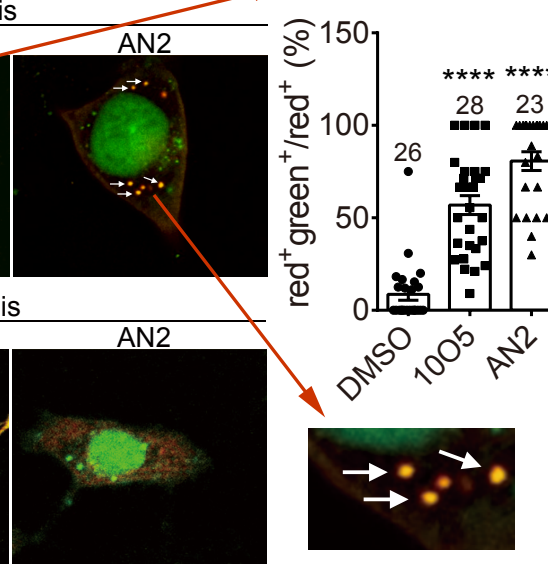

d

GFP-LC3B+Q25-His
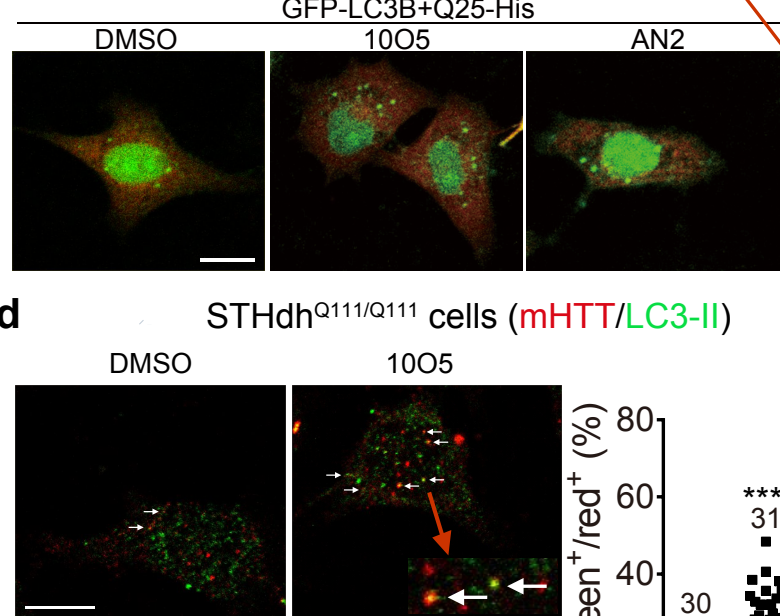

AN2

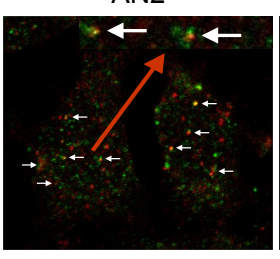

HTT SiRNA
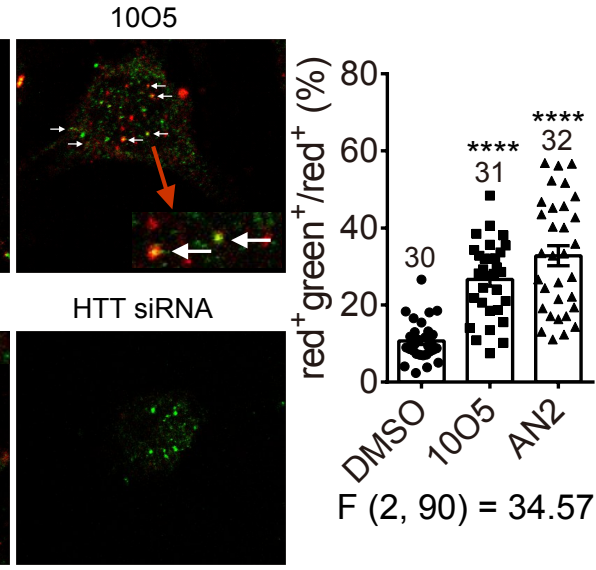

anti-GST GST- 


\section{Figure 5}

a

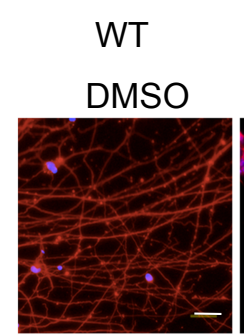

b Human iPSC-derived neurons (-BDNF at $0 \mathrm{~h}$ ) c

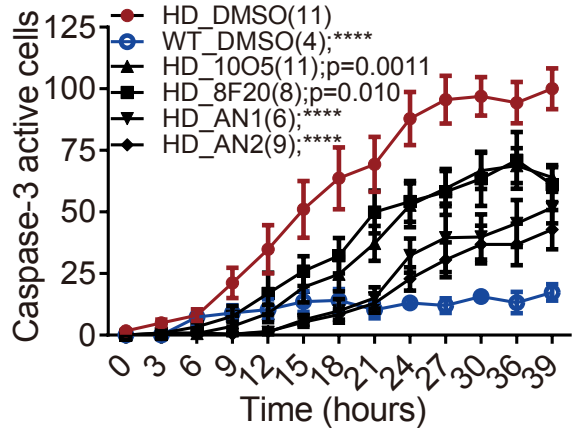

d

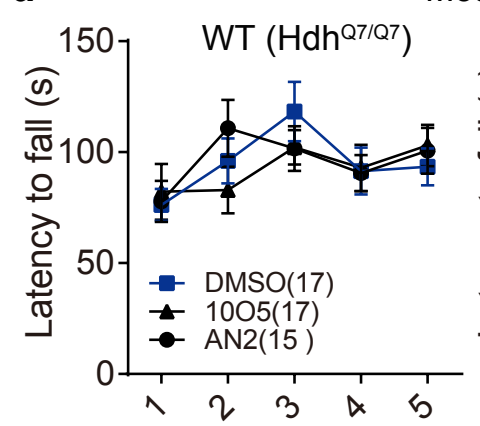

Days

Mouse gripping force tests

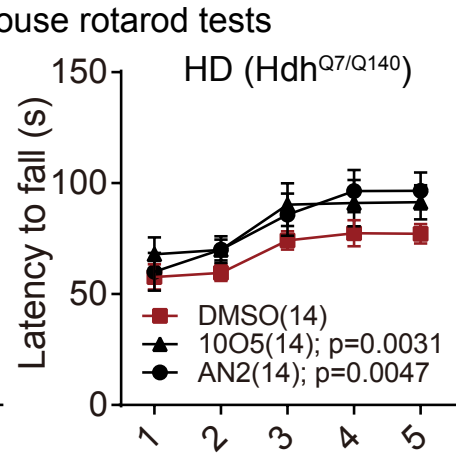

Days

$\mathbf{f}$

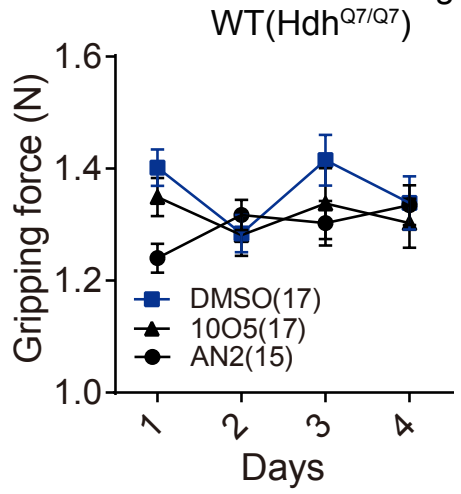

Mouse rotarod tests

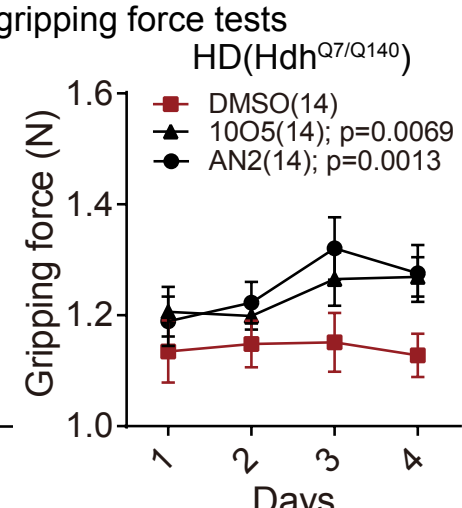

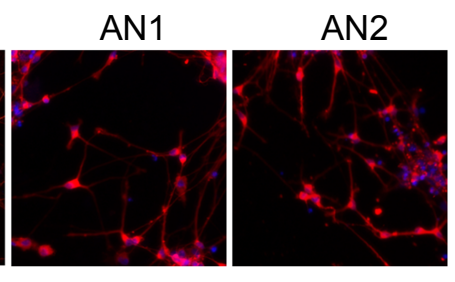

Drosophila models expressing human full-length HTT

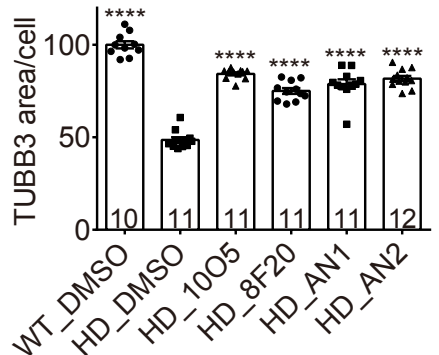

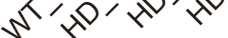
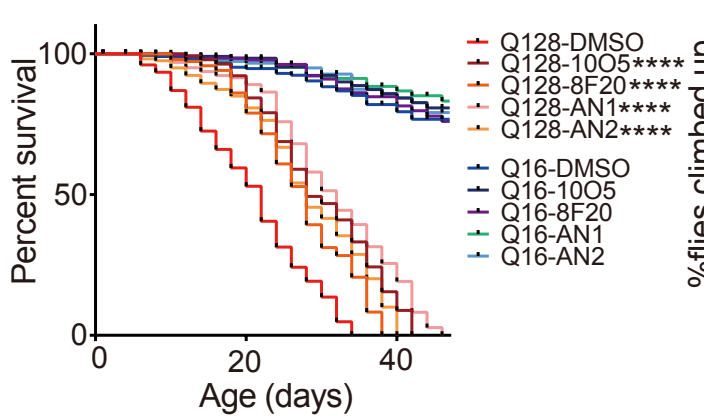

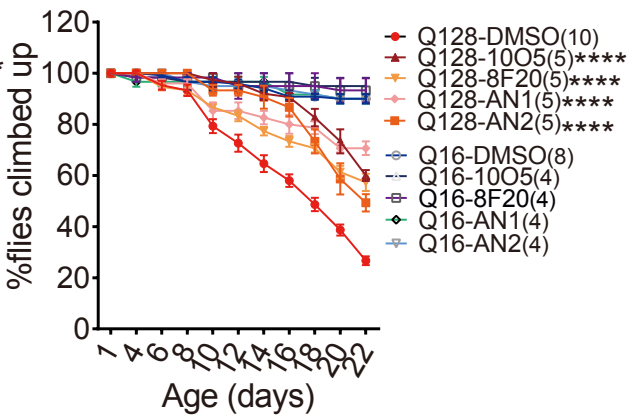

Mouse balance-beam tests

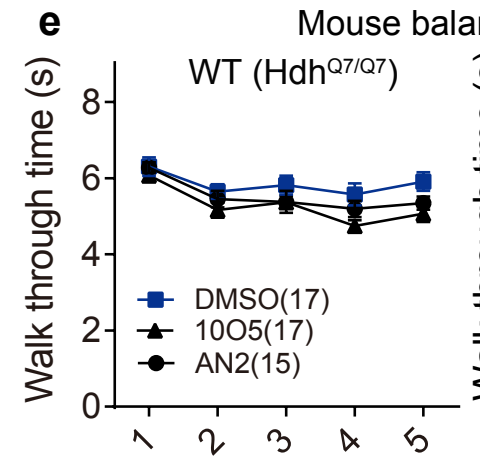

Days g

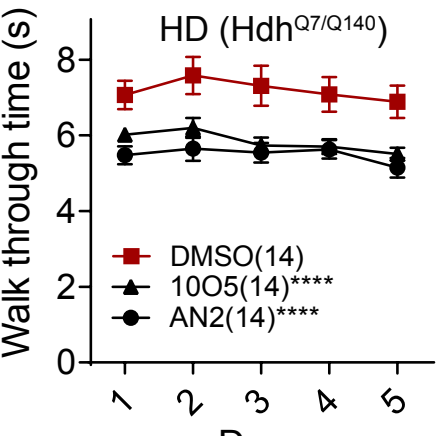

Days
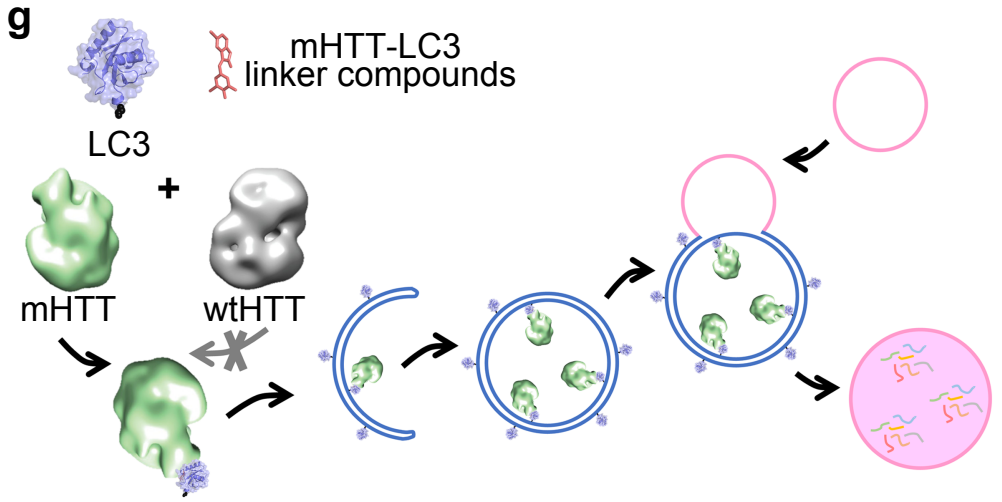

mHTT-LC3 phagophore autophagosome autolysosome 